\title{
The Face-Processing Network Is Resilient to Focal Resection of Human Visual Cortex
}

\author{
○Kevin S. Weiner, ${ }^{1}$ Jacques Jonas, ${ }^{2,3,4}$ Jesse Gomez, ${ }^{5}$ Louis Maillard, ${ }^{2,4}$ Hélène Brissart, ${ }^{2}$ Gabriela Hossu, ${ }^{6}$

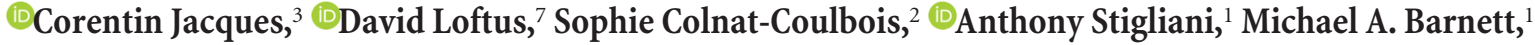 \\ Kalanit Grill-Spector, ${ }^{1,5 *}$ and Bruno Rossion ${ }^{3 *}$ \\ ${ }^{1}$ Department of Psychology, Stanford University, Stanford, California 94305, 2Department of Neurology and Neurosurgery, University Hospital of Nancy, \\ 54000 Nancy, France, ${ }^{3}$ Psychological Science Research Institute and Institute of Neuroscience, University of Louvain, 1348 Louvain-la-Neuve, Belgium, \\ ${ }^{4}$ CRAN, UMR 7039, CNRS and University of Lorraine, 54000 Nancy, France, ${ }^{5}$ Neurosciences Institute, Stanford University, Stanford, California 94305 , \\ ${ }^{6}$ Clinical Investigation Centre Innovative Technology, University Hospital of Nancy, 54000 Nancy, France, and 7Department of Psychology, Pomona College, \\ Claremont, California 91711
}

Human face perception requires a network of brain regions distributed throughout the occipital and temporal lobes with a right hemisphere advantage. Present theories consider this network as either a processing hierarchy beginning with the inferior occipital gyrus (occipital face area; IOG-faces/OFA) or a multiple-route network with nonhierarchical components. The former predicts that removing IOG-faces/OFA will detrimentally affect downstream stages, whereas the latter does not. We tested this prediction in a human patient (Patient S.P.) requiring removal of the right inferior occipital cortex, including IOG-faces/OFA. We acquired multiple fMRI measurements in Patient S.P. before and after a preplanned surgery and multiple measurements in typical controls, enabling both within-subject/ across-session comparisons (Patient S.P. before resection vs Patient S.P. after resection) and between-subject/across-session comparisons (Patient S.P. vs controls). We found that the spatial topology and selectivity of downstream ipsilateral face-selective regions were stable 1 and 8 month(s) after surgery. Additionally, the reliability of distributed patterns of face selectivity in Patient S.P. before versus after resection was not different from across-session reliability in controls. Nevertheless, postoperatively, representations of visual space were typical in dorsal face-selective regions but atypical in ventral face-selective regions and $\mathrm{V} 1$ of the resected hemisphere. Diffusion weighted imaging in Patient S.P. and controls identifies white matter tracts connecting retinotopic areas to downstream face-selective regions, which may contribute to the stable and plastic features of the face network in Patient S.P. after surgery. Together, our results support a multiple-route network of face processing with nonhierarchical components and shed light on stable and plastic features of high-level visual cortex following focal brain damage.

Key words: brain lesion; cortical plasticity; face perception; fusiform face area; hierarchical networks; occipital face area

\section{Significance Statement}

Brain networks consist of interconnected functional regions commonly organized in processing hierarchies. Prevailing theories predict that damage to the input of the hierarchy will detrimentally affect later stages. We tested this prediction with multiple brain measurements in a rare human patient requiring surgical removal of the putative input to a network processing faces. Surprisingly, the spatial topology and selectivity of downstream face-selective regions are stable after surgery. Nevertheless, representations of visual space were typical in dorsal face-selective regions but atypical in ventral face-selective regions and V1. White matter connections from outside the face network may support these stable and plastic features. As processing hierarchies are ubiquitous in biological and nonbiological systems, our results have pervasive implications for understanding the construction of resilient networks.

\section{Introduction}

Visual recognition requires a series of processing stages along the ventral visual pathway of the human brain ascending from primary visual cortex to occipitotemporal cortex (Ungerleider and Mishkin, 1982; Felleman and Van Essen, 1991; Kravitz et al., 2013). Understanding the structural-functional organization of this pathway is a major neuroscientific goal. Human face perception is of unique interest to achieve this goal because faces are the most socially meaningful stimuli in our environment; and as such, face perception recruits a series of cortical regions responding more strongly to faces than nonfaces extending from lateral occipital cortex to ventral aspects of the temporal lobe, with a 
right hemisphere dominance (Sergent et al., 1992; Puce et al., 1995; Haxby et al., 2000; Fox et al., 2008; Rossion, 2008; Pitcher et al., 2011a; Zhen et al., 2013; Duchaine and Yovel, 2015). A subset of these regions composes the "core" face network (Haxby et al., 2000): the inferior occipital gyrus/occipital face area (IOG-faces/ OFA) (Gauthier et al., 2000; Weiner and Grill-Spector, 2010), the fusiform gyrus (FG)/fusiform face area (FFA) (Kanwisher et al., 1997), which can be divided into separate regions on the posterior and mid-fusiform gyrus (Pinsk et al., 2009; Weiner and GrillSpector, 2010), and regions along the superior temporal sulcus (STS) (Puce et al., 1998; Pinsk et al., 2009; Pitcher et al., 2011b). The prevailing neurofunctional model of face perception (Haxby et al., 2000; Fairhall and Ishai, 2007) proposes that this "core" face network is organized hierarchically, which suggests that neural computations in regions of the face network follow and depend on computations performed by what is considered the input of the entire network: IOG-faces/OFA.

However, the hierarchical model of the face network has been questioned by studies showing face-selective responses within the FG and STS, even in the absence of face-selective responses in the IOG, both in participants with unilateral or bilateral lesions to the IOG (Rossion et al., 2003; Steeves et al., 2006) and healthy participants (Rossion et al., 2011; Pitcher et al., 2014). These findings have inspired alternative nonhierarchical models of the face network (Rossion, 2008; Atkinson and Adolphs, 2011; Pitcher et al., 2011a; Duchaine and Yovel, 2015; Yang et al., 2016), suggesting that information can reach more anterior regions of the face network without the IOG. Although fMRI studies in patients with long-term brain damage provide causal support for these nonhierarchical proposals, functional measurements in these patients are conducted years after the damage has occurred, with no baseline measurement before the lesion. Consequently, a putative normative state of the functional region or network of interest before damage is assumed rather than known. Further, it is impossible to know whether any reorganization has occurred during the prolonged time interval between the damage and eventual measurement. An appealing alternative to directly test hierarchical and nonhierarchical models of the face network is a functional neuroanatomical investigation of the face network before and after surgical removal of the right IOG. However, this approach is largely impossible in humans due to the rarity of fMRI measurements before a preplanned surgery (Gaillard et al., 2006).

Here, we present a unique case in which a patient (Patient S.P.) required focal resection of the right IOG, including IOGfaces/OFA. We examined the functional organization of the cortical face network before and after removal of the right IOG using numerous methods: (1) multiple sessions of fMRI, (2) intracerebral electrophysiology (iEEG), (3) neuropsychological testing, and (4) diffusion weighted imaging (DWI) with tractography.

Received Dec. 16, 2015; revised June 21, 2016; accepted June 22, 2016.

Author contributions: K.S.W., J.J., L.M., K.G.-S., and B.R. designed research; K.S.W., J.J., L.M., H.B., G.H., C.J., A.S., M.A.B., K.G.-S., and B.R. performed research; K.S.W., J.J., J.G., C.J., S.C.-C., A.S., M.A.B., K.G.-S., and B.R. contributed unpublished reagents/analytic tools; K.S.W., J.J., J.G., C.J., D.L., A.S., M.A.B., K.G.-S., and B.R. analyzed data; K.S.W., J.J., K.G.-S., and B.R. wrote the paper.

This work was supported by National Institutes of Health 1R01 EY 02391501A1 to K.G.-S., ERC Grant facessvep 284025 to B.R., the Belgian National Fund for Scientific Research, and Belgian Science Policy Office.

The authors declare no competing financial interests.

*K.G.-S. and B.R. contributed equally to this study.

Correspondence should be addressed to Dr. Kevin S. Weiner, Department of Psychology, Stanford University, Stanford, CA 94305. E-mail: kweiner@stanford.edu.

DOI:10.1523/JNEUROSCI.4509-15.2016

Copyright $\odot 2016$ the authors $\quad 0270-6474 / 16 / 368426-16 \$ 15.00 / 0$
Volume anatomy
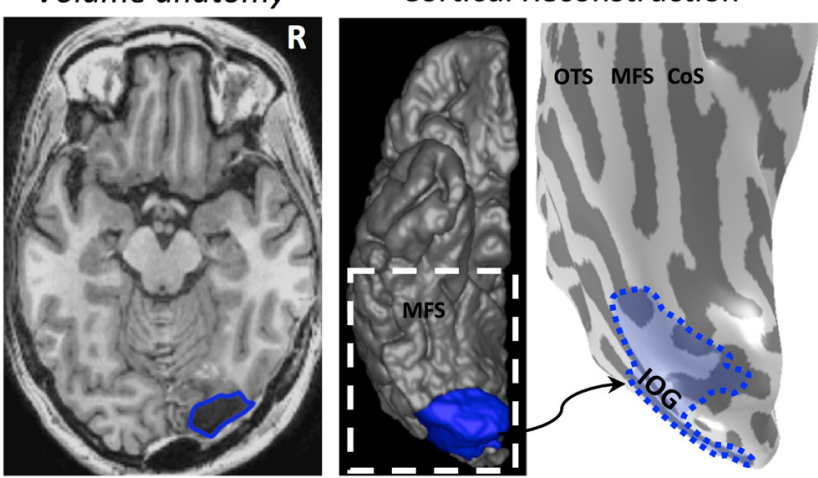

Figure 1. Reconstructing the resection. The resection (blue) included the $10 \mathrm{G}$ and surrounding cortex. After aligning the preoperative and postoperative anatomies, we projected the resected tissue onto the presurgical anatomy. All analyses were done on the pre-resection anatomy. Left, Axial slice of the post-resection T1. Middle, Reconstruction of the postoperative cortical surface and resected tissue (blue). White dotted line indicates the zoomed portion in the rightmost image. Right, Inflated preoperative cortical surface with the aligned resected cortex shaded in blue. CoS, Collateral sulcus; MFS, mid-fusiform sulcus; OTS, occipitotemporal sulcus.

This combinatorial approach directly examines how physically removing the putative input to a cortical hierarchy in the living human brain affects the neurofunctional organization of downstream regions compared with the original state of the network, as well as presents a unique opportunity to causally test the hierarchical and nonhierarchical models of the face network. The former predicts a detrimental effect of resection on downstream face-selective regions, whereas the latter predicts resiliency of downstream face-selective responses despite the removal of right IOG-faces/OFA.

\section{Materials and Methods}

\section{Patient S.P.}

Patient S.P. is a 36-year-old right-handed woman who had refractory epilepsy related to a right benign dysembryoplastic neuroepithelial tumor. The tumor involved the right occipital pole and extended to the infracalcarine region. Patient S.P. is a high-functioning patient: (1) before resection, she had an IQ of 98, her visual field was normal, and her scores on the Visual Object and Space Perception battery assessing basic visual functions (Warrington and James, 1991) were maximal for all 8 subtests; (2) she works as a pharmacist and has a high level of education (baccalauréat +6 in France, totaling 18 years of studies since she was 6 years old); and (3) as far as we know, she has a typical social life, interacting with her family, colleagues, and friends.

The iEEG delineated the seizure onset zone within the tissue related to the tumor. The epileptic onset zone did not involve IOG-faces/OFA. Anatomically, surgical resection involved the tumor zone and surrounding cortex, including the IOG, posterior FG, and portions of the lateral occipitotemporal cortex (Fig. 1). Two years after the operation, the patient remains seizure-free. Written consent was obtained from Patient S.P., and the procedures were approved by the local ethical committee of the University Hospital of Nancy for clinical protocols.

\section{fMRI}

fMRI data acquisition

Exclusive to our case, we acquired multiple fMRI sessions before and after resection to distinguish the effect of resection from session-tosession variability. Patient S.P. participated in five fMRI sessions (S1, S2, S3, S4, and S5) on different days. S1 and S2 were presurgical. S3, S4, and S5 were postsurgical. S1 and S2 were 30 and $4 \mathrm{~d}$ before surgery, respectively. S3, S4, and S5 were $30 \mathrm{~d}, 8$ months, and 18 months after surgery, respectively. In S1, S2, S3, and S4, Patient S.P. participated in multiple runs of a block design experiment designed to measure responses to different visual categories. In S1, Patient S.P. also participated in a stan- 
dard retinotopic mapping experiment, as well as an event-related experiment using images of faces, cars, and their phase-scrambled counterparts. In S5, Patient S.P. participated in an experiment during which images of faces, limbs, and houses appeared at fixation or $3^{\circ}$ to the right or left of fixation (3 position experiment).

All functional and anatomical imaging sessions were performed on the same 3 Tesla GE scanner (Signa HDXT, GE Medical Systems) at the University Hospital of Nancy (Nancy, France) using an 8-channel head coil. In S1, we acquired 38 slices at a resolution of $3.75 \times 3.75 \times 3.5 \mathrm{~mm}$ using a standard EPI sequence $(\mathrm{FOV}=240 \mathrm{~mm}, \mathrm{TE}=35 \mathrm{~ms}, \mathrm{TR}=2000$ ms, flip angle $=90^{\circ}$ ). In S2-S5, we acquired 36 slices at a resolution of $3 \times$ $3 \times 3 \mathrm{~mm}$ using a standard EPI sequence $(\mathrm{FOV}=192 \mathrm{~mm}, \mathrm{TE}=33 \mathrm{~ms}$, $\mathrm{TR}=2000 \mathrm{~ms}$, flip angle $\left.=77^{\circ}\right)$. In S2-S5, inplane anatomicals were also acquired with the same slice prescription as the functionals using a T1weighted SPGR pulse sequence $(\mathrm{FOV}=192 \mathrm{~mm}$, TE $=$ minimum, flip angle $=25^{\circ}$ ). In all sessions, high-resolution anatomical volumes of the whole brain were acquired using a T1-weighted SPGR pulse sequence (resolution: $1 \times 1 \times 1 \mathrm{~mm}$ ), which was used to create reconstructions of the cortical surface.

\section{fMRI experiments}

Localizer, block experiment. Patient S.P. participated in 3 runs of this experiment in S1, 2 runs in S2, and 2 runs in two of the postsurgical scanning sessions (S3, S4). During the fMRI scan, she viewed images of faces, limbs, places, objects, and phase-scrambled images. Exemplars appeared in variable viewing conditions and were not repeated across runs. Each run was $288 \mathrm{~s}$ long, consisted of randomized $12 \mathrm{~s}$ blocks of each condition ( 3 per condition), and contained 8 blank blocks. Each run began and ended with $12 \mathrm{~s}$ of blank. Patient S.P. performed a 1-back task as in our prior experiments (Weiner and Grill-Spector, 2010, 2011, 2013; Weiner et al., 2014).

Localizer, event-related experiment. Patient S.P. participated in 3 runs of this experiment in presurgical S1. Images of faces, cars, and phase-scrambled images were presented for $2.25 \mathrm{~s}$, followed by a 7.75-11.25 s blank interval. Each run lasted $504 \mathrm{~s}$, during which 11 images of each type (44 in total) were presented. Images were not repeated within or across runs.

Traveling wave retinotopy experiment. Patient S.P. participated in standard retinotopic mapping (Engel et al., 1997) in S1. While fixating, she participated in 2 runs during which she viewed rotating black-and-white checkerboard wedges or expanding checkerboard rings. Patient S.P. was instructed to fixate on a central cross and respond by button press when the fixation cross changed colors.

Three position experiment. During S5, Patient S.P. participated in an experiment designed to measure fMRI responses to contralateral and ipsilateral stimuli. During fMRI, images of faces, houses, and limbs appeared in three positions: (1) at fixation, (2) $3^{\circ}$ of visual angle to the left of fixation, and (3) $3^{\circ}$ of visual angle to the right of fixation. Exemplars appeared in variable viewing conditions within $4 \mathrm{~s}$ blocks for 3 runs. Each run was $200 \mathrm{~s}$ long, consisted of randomized blocks of each condition ( 4 per condition), and contained 12 blank blocks. Each run also began and ended with $4 \mathrm{~s}$ of a blank screen. Patient S.P. was instructed to fixate on a central cross and respond by button press when the fixation changed color.

\section{fMRI data analysis}

Data were analyzed with MATLAB (The MathWorks) using the mrVista toolbox (http://white.stanford.edu/software). Presurgical and postsurgical T1 scans were aligned to the AC-PC plane. Automated (Dale et al., 1999) (FreeSurfer: http://surfer.nmr.mgh.harvard.edu) and manual (ITK-SNAP: http://www.itksnap.org/pmwiki/pmwiki.php) segmentation tools were used to segment gray and white matter, from which we reconstructed the cortical surface (Wandell et al., 2000). For visualization, data were projected to the presurgical anatomy after aligning presurgical and postsurgical volumes and determining resection boundaries from postsurgical scans (Fig. 1). Functional data of each session were motion corrected and temporally high-pass filtered with $1 / 20 \mathrm{~Hz}$ cutoff and converted to percentage signal change. Response amplitudes were estimated using a GLM applied to the time-series of each voxel using as predictors the experimental conditions convolved with the hemodynamic impulse response function used in SPM. Data were not spatially smoothed. A GLM was also used to generate $t$ maps of selectivity showing significantly higher responses to one condition versus others.

Definition of face-selective regions of interest (ROIs). Four face-selective clusters were defined in the right hemisphere of Patient S.P. from the localizer experiment from 1 run in S1 with a contrast of faces $>$ limbs, places, and objects ( $t>3$, voxel level) as in our prior studies (Weiner and Grill-Spector, 2010, 2013): (1) posterior fusiform (pFus)-faces/FFA-1, (2) IOG-faces/OFA, (3) posterior superior temporal sulcus (pSTS)-faces, and (4) mid superior temporal sulcus (mSTS)-faces. In the left hemisphere, we were able to identify mFus-faces/FFA-2, pFus-faces/FFA-1, and pSTS-faces in all sessions, but not the left IOG-faces/OFA in any session. We compared the localization of these activations before and after resection. Data from right hemisphere are presented in Figures 3-5, and 7, and data from the left hemisphere in Figures 6 and 7.

We were able to identify two additional regions in the right hemisphere in a subset of the sessions. Specifically, we were able to identify mFus-faces/FFA-2 (Weiner and Grill-Spector, 2010) only in one of the pre-resection sessions (Fig. 4). To test whether this was a measurability problem due to the ear canal artifact, we measured the time course signalto-noise ratio (tSNR) in each run. A tSNR threshold of 35 is necessary to measure a statistically meaningful result given our resolution, magnetic strength, and scan duration (Murphy et al., 2007). However, in 3 of the 4 independent pre-resection runs in mFus-faces, tSNR was not significantly higher than the required tSNR for measurability (all $p$ values $>0.29$ ). This is not specific to Patient S.P. as we have previously shown that MR artifacts induced by the ear canal can affect the measurability and tSNR of mFus-faces/FFA-2 (Weiner and Grill-Spector, 2013). Because mFus-faces/FFA-2 is unstable before resection, we do not include this region in our subsequent analyses. It is also important to reiterate that we can identify this region in Patient S.P. in the left hemisphere; this is because MR artifacts are not subject-specific, but hemisphere-specific, and can differentially affect the measurability of the same region across hemispheres (Weiner and Grill-Spector, 2013). Indeed, in Patient S.P., whereas the tSNR is low in right mFus-faces/FFA-2 across sessions as reported above, it is stable and $>35$ across sessions in left mFus-faces/ FFA-2 (pre-resection tSNR mean \pm SD: $102 \pm 59$; post-resection tSNR: $105 \pm 28$; these values are not significantly different, $p=0.93$, paired $t$ test).

We were also able to identify an additional region on the inferior occipital sulcus (IOS) in both pre-resection sessions (see Fig. 4). However, this region is typically not included in neurocognitive models of face perception as it does not respond specifically to faces but responds to animate stimuli more generally, as well as overlaps with nearby bodyselective regions (Weiner and Grill-Spector, 2013). Indeed, responses to faces and bodies within the IOS were not significantly different in Patient S.P. (before resection, $p=0.60$ ) or in a group of typical controls $(N=10$, $p=0.70$; see $\mathrm{fMRI}$ and diffusion weighted imaging in control subjects).

Mean response amplitudes and selectivity. Analyses of mean response amplitudes and selectivity were conducted on data that were independent from those used to define the ROIs. For the former, we first calculated mean response amplitudes estimated from GLM betas in each voxel and then averaged these responses across voxels in each ROI. For the latter, we first calculated face selectivity within each voxel as faces $>$ other stimuli and then calculated the mean selectivity across voxels of each ROI within each run of the experimental session (see Figs. 3, 4, 6). Importantly, all analyses within right pFus-faces excluded resected portions to assure that comparisons before and after resection included the same voxels.

Reliability of face selectivity across voxels. To examine the reliability of face selectivity across voxels, we calculated the distributed face selectivity in each ROI for each run and then calculated the correlation $(r)$ between distributed face selectivity of the ROI across sessions. We calculated these values three different ways in Patient S.P.: (1) between experimental runs across pre-resection sessions (see Fig. 5, red); (2) between experimental runs across post-resection sessions (see Fig. 5, black); and (3) between experimental runs across pre-resection and post-resection sessions (see 
A

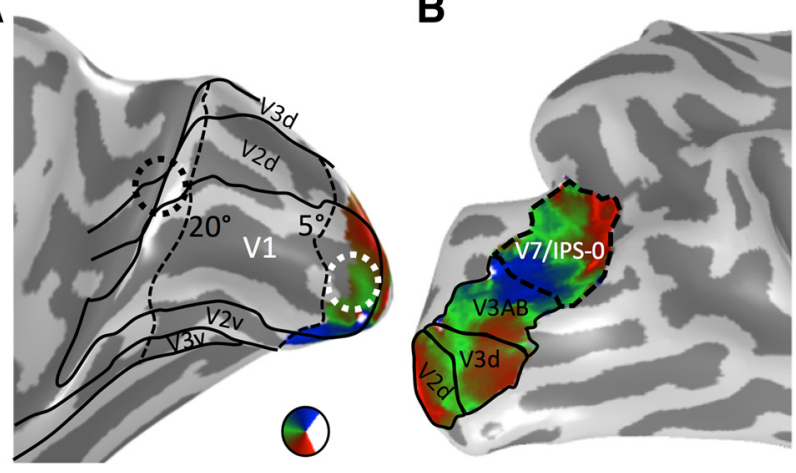

Figure 2. Retinotopic ROls. $\boldsymbol{A}$, The polar angle map of $\mathrm{V} 1$ on the medial inflated cortical surface of Patient S.P. before resection. Our fMRI scanning setup allowed us to map eccentricities out to $5^{\circ}$. Representations of the far periphery $\left(>20^{\circ}\right)$ were defined from the cortical sheet using recently published tools (Benson et al., 2014). We defined two ROls for white matter tracking: the central $5^{\circ}$ of $\mathrm{V} 1$ (white circle) and eccentricities outside the postsurgery scotoma (beyond $20^{\circ}$, black circle). $B$, Polar angle maps from V2d to V7/IPS-0 on the lateral inflated cortical surface of Patient S.P. before resection. V7/IPS-0 (dashed line) was used for identifying potential vertical white matter tracts reaching pFus-faces.

Fig. 5, blue). We calculated these reliability metrics for right pFus-faces, pSTS-faces, and mSTS-faces.

Definition of retinotopic ROIs. We delineated retinotopic visual areas V1-V7/IPS-0 from the traveling wave retinotopic experiment conducted before resection in S1 (Fig. 2). Because of limitations of the scanner setup, we could only map visual field representations within the central $5^{\circ}$. V1 was used for examining visual field representations in the three position experiment, and to define ROIs for DWI tracking of white matter tracts between early visual cortex to downstream face-selective regions. V7/ IPS-0 ROI was defined for white matter tracking of vertical tracts to the fusiform. Additionally, we used cortex-based alignment techniques to align templates of early visual regions V1-V3 (Benson et al., 2012, 2014) to Patient S.P.'s cortical surface to delineate the entire eccentricity representation of these regions (Fig. 2A).

Visual field preference ( 3 position experiment). Within V1, pFus-faces, mSTS-faces, and pSTS-faces, we calculated the visual field preference in response to stimuli presented in the right and left visual field in each voxel by calculating the $t$ value comparing responses to stimuli in the left versus right visual field collapsed across categories. Negative numbers indicate preference for the left visual field (expected for right hemisphere ROIs), and positive numbers indicate preference for the right visual field (expected for left hemisphere ROIs). We report the distribution of these visual field preferences across all voxels of an ROI separately for the right, resected hemisphere and the left, nonresected hemisphere. We assessed differences in visual field preferences in the left versus right ROIs using a Kolmogorov-Smirnov test.

\section{DWI}

Data acquisition. We acquired DWI in S1, S3, and S4 using a diffusionweighted, single-shot echo-planar imaging sequence: presurgery/postsurgery S1 and S3 parameters: 25 diffusion directions, $\mathrm{b}=1000 \mathrm{~s} / \mathrm{mm}^{2}$, resolution of $2 \times 2 \times 2 \mathrm{~mm}$; postsurgery $\mathrm{S} 4$ parameters: 64 diffusion directions, $\mathrm{b}=2000 \mathrm{~s} / \mathrm{mm}^{2}$, resolution $2 \times 2 \times 2.6 \mathrm{~mm}$.

Data analysis. Eddy-current distortions and subject motion were corrected and removed using a constrained 14-parameter nonlinear coregistration method according to the eddy-current distortions created by the specific phase-encoded direction of the acquired data. Diffusionweighted images were registered to the non-diffusion-weighted (b0) image using a two-stage coarse-to-fine approach to maximize mutual information. The $\mathrm{b} 0$ image was aligned to the $\mathrm{T} 1$ image with a rigid body algorithm. All processing was performed using Stanford University's open-source mrDiffusion package (http://white.stanford.edu/software).

We performed whole-brain white matter tractography using MRtrix software (Tournier et al., 2007, 2012). Each white matter voxel was used as a seed of origin for fiber tracking, and a constrained-spherical decon- volution (as in Gomez et al., 2015) model of diffusion was fit at every voxel within white matter. Fibers were traced within a white matter mask using the probabilistic tractography implementation within MRtrix using 600,000 seedpoints and a maximum harmonic envelope of 4 was used to model crossing fibers.

Candidate white matter bundles were extracted by intersecting the optimized connectome with volume ROIs. ROIs were constructed by dilating each functional ROI in each subject's native brain space, with a 3 -voxel smoothing kernel to encompass the neighboring white matter voxels. Candidate pathways interconnecting two ROIs were extracted from the whole brain connectome through Boolean 'AND' operations.

Examining white matter tracts between early visual areas and faceselective regions. Recent results show that face-selective regions on the IOG and STS are structurally connected to the calcarine sulcus via longitudinal white matter tracts (Gschwind et al., 2012). However, it is likely that these tracts originate separately from the foveal and peripheral representations of early visual areas. Indeed, nonhuman primates have anatomical connections from the periphery of V1/V2 to the periphery of MT (Desimone and Ungerleider, 1986). It is feasible that STS faceselective regions may be structurally connected to the periphery of early visual areas as these regions are adjacent to the periphery of MT in humans (Weiner and Grill-Spector, 2013). Thus, we examined both sets of longitudinal white matter tracts connecting (1) an ROI within the central $5^{\circ}$ of V1 (Fig. 2A) and ventral face-selective regions (IOG-faces and pFusfaces, respectively) and (2) an ROI overlapping eccentricities $>20^{\circ}$ of early visual areas (Fig. 2A) and STS face-selective regions (pSTS-faces and mSTS-faces). For tracking, we refer to these ROIs as V1/V2 because growing these ROIs as spheres into white matter likely encroached into portions of $\mathrm{V} 2$.

Recent results also show that the posterior fusiform is connected to dorsal retinotopic regions via vertical white matter tracts (Kim et al., 2006; Takemura et al., 2016). As the latter study revealed that these tracts are part of the vertical occipital fasciculus (VOF) (Yeatman et al., 2014; Takemura et al., 2016; Weiner et al., 2016) and connect hV4 ventrally to retinotopic maps dorsally extending to V7/IPS-0, we also examined whether portions of the VOF connect V7/IPS-0 (Fig. 2B) to pFus-faces. The VOF was defined by including all fibers terminating in posterior aspects of ventral occipitotemporal cortex with a vertical to horizontal length ratio of $\geq 1.3$ (Yeatman et al., 2014).

Mapping fiber endpoints to the resected volume. It is difficult to acquire DWI data following stroke or cortical damage due to artifacts in the diffusion signal as a result of increased diffusivity surrounding the damage (Pasternak et al., 2009). Consistent with these prior reports, the resected tissue generated significant artifacts in and around the resection, which prevented us from being able to accurately track longitudinal fiber tracts to the FG in post-resection scans S3 and S4. Despite these difficulties, to gain clarity regarding how connectivity of the face network is affected by the loss of connections associated with IOG-faces/OFA, we mapped the pre-resection cortical endpoints of the tracts from early visual cortex to either IOG-faces/OFA or pFus-faces/FFA-1 to the postresection volume (see Fig. 8). In contrast to the artifacts affecting ventral longitudinal tracts, the resection did not affect DWI signals in dorsal longitudinal tracts or vertical tracts in Patient S.P. (see Figs. 9, 10).

\section{iEEG}

IEEG recording was performed to identify the epileptogenic zone, using intracerebral electrodes (stereo-EEG) (Bancaud and Talairach, 1973). The iEEG was performed once, 6 months before resection. Electrode implantation sites were chosen according to noninvasive data collected during the earlier phase of the investigation to localize and delineate the zone of epileptic seizure onset and early propagation. Nine electrodes were placed in the right hemisphere targeting the hippocampus, the rhinal cortex, the ventral occipitotemporal cortex (lingual gyrus, collateral sulcus, fusiform gyrus, and occipitotemporal sulcus), the occipital pole, the calcarine sulcus, and the occipitoparietal junction. One electrode was placed in the left hemisphere, in the middle ventral temporal region. Postimplant CT images were aligned to the preoperative MRI anatomical brain volume (Hermes et al., 2010). Electrodes were visualized on the subject's own 
A

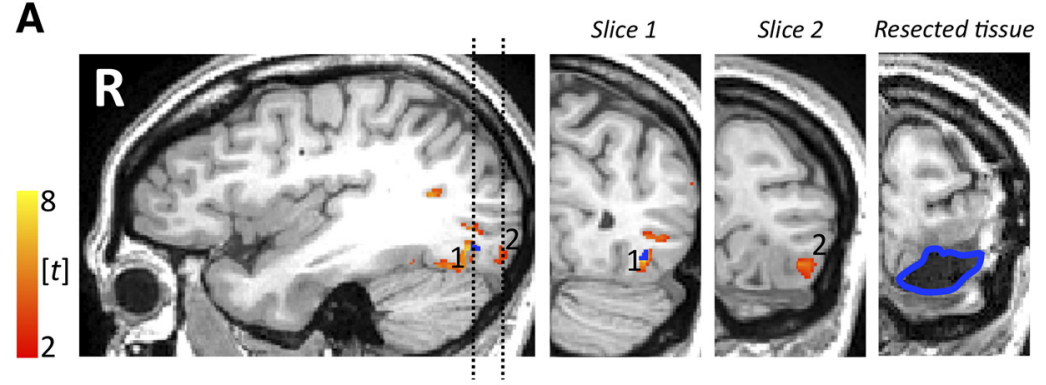

C

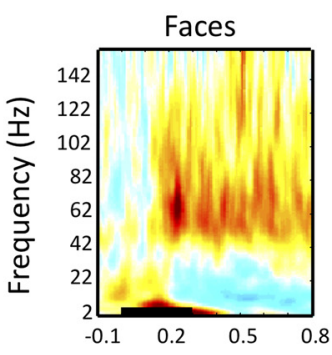

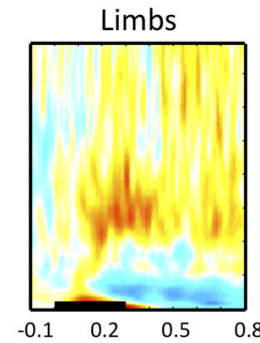

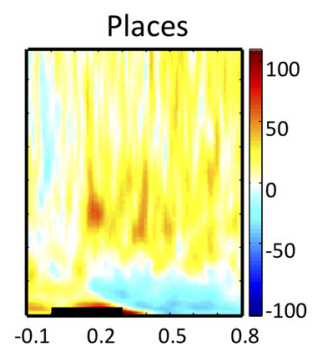

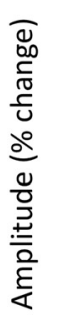

B

\section{pFus-faces/FFA-1 IOG-faces/OFA electrode}

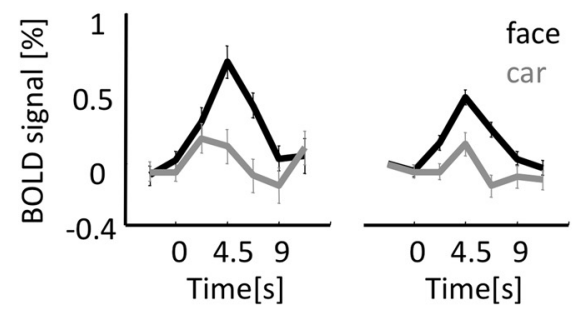

D

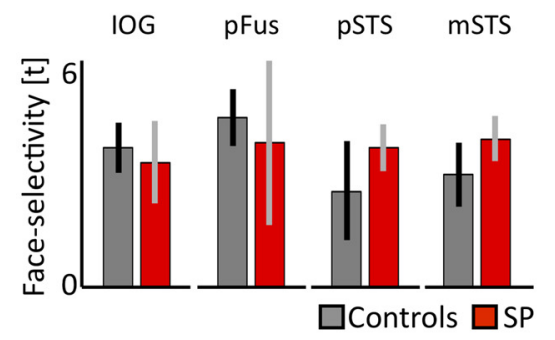

E

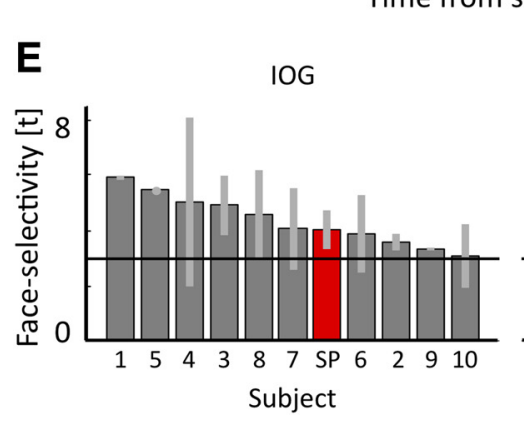
IOG

Time from stimulus onset [s]
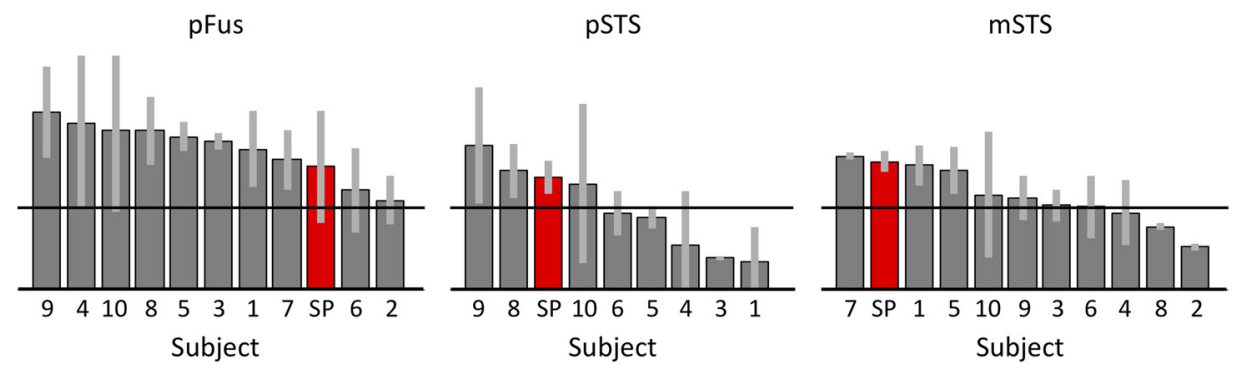

Figure 3. The face network is typical before surgery. $\boldsymbol{A}$, Regions illustrating face-selective responses ( $t>3$ ) before surgery. Blue represents intracerebral electrode within pFus-faces/FFA-1. Dotted lines indicate location of the two coronal slices shown on the right two panels. 1, pFus-faces/FFA-1; 2, I0G-faces/OFA. Blue outline indicates resected tissue on post-resection anatomy in similar position as slice at left. $\boldsymbol{B}$, fMRI response under electrode and from IOG-faces/OFA from an independent experiment. Error bars indicate SEM across runs. $\boldsymbol{C}$, Time-frequency responses of pFus-faces/FFA-1 electrode. Signal amplitude (\% change from baseline) is averaged across trials and category. Black line indicates stimulus $0 \mathrm{~N}$ window (300 ms). High-frequency broadband responses showed significantly higher $(p<0.005)$ power to faces compared with limbs or places $150 \mathrm{~ms}$ after stimulus onset. $\boldsymbol{D}$, Mean face selectivity in controls (gray) and Patient S.P. (red) in the IOG $(N=10)$, pFus $(N=10)$, pSTS $(N=8)$, and $\mathrm{mSTS}(N=10)$. Error bars for controls indicate mean and SD between runs averaged across subjects. Error bars for Patient S.P. indicate SD across 4 independent pre-resection runs. $E$, Mean and SD of face selectivity in individual controls (gray) and Patient S.P. (red) in the IOG $(N=10)$, pFus $(N=10)$, pSTS $(N=8)$, and mSTS $(N=10)$. Each bar indicates data from one participant. The $x$-axis is ranked in a descending order to indicate where Patient S.P. falls in the distribution of typical participants. Error bars indicate SD between runs. Solid black line indicates the statistical threshold $(t>3)$ used to define each region from independent data.

brain volume and reconstructed 3D cortical surface allowing for accurate anatomical localization of electrodes (Fig. 3).

Category iEEG experiment. Patient S.P. viewed 50 black-and-white pictures of faces, body parts, places, and phase-scrambled faces in the hospital room at a distance of $70 \mathrm{~cm}$ from a computer monitor. In each trial, a stimulus was displayed at the center of the monitor for $300 \mathrm{~ms}$, followed by an average intertrial interval of $1750 \mathrm{~ms}$ (1450-2050 ms). The patient fixated on a central cross and pressed a key when a face image was presented upside-down ( $9 \%$ of trials). Patient S.P. performed 4 blocks of 77 trials with conditions randomized within each block.

Data acquisition and analysis. The iEEG signal was recorded at $512 \mathrm{kHz}$ using a 128-channel amplifier (2 SD LTM 64 Headbox; Micromed) relative to a prefrontal scalp reference electrode. Continuous EEG data were notch-filtered to remove line noise $(50 \mathrm{~Hz}$ and harmonics) and segmented in -1.8 to $1.8 \mathrm{~s}$ epochs centered on the onset of each trial. Noisy trials in which signal amplitude in a -0.6 to $0.4 \mathrm{~s}$ time-window was $>3.3$ or $<3.3$ times the across-trial SD were discarded. The signal at each electrode was then rereferenced to the average of all intracranial electrodes. Variations in signal amplitude as a function of time and frequency were estimated using a Morlet wavelet approach as in prior studies (Jacques et al., 2016). Face selectivity was tested by comparing the response amplitude in the high-frequency broadband range $(40-100 \mathrm{~Hz})$ elicited by face images compared with place and body part images. Statistical significance was calculated using permutation tests at each time sample of the response between -0.1 and 0.8 s relative to stimulus onset. This process was performed 10,000 times to generate a distribution. Differences at a $p<0.01$ (two-tailed) that lasted for at least 4 consecutive time samples were considered significant.

\section{Neuropsychological assessment}

We conducted a stringent battery of behavioral tests on subject Patient S.P. before (11 months) and after (1 month) cortical resection to assess her general cognitive function, basic visual perception, as well as face and object perception and recognition. These tests included full-scale IQ testing (WAIS-IV), basic visual perception testing (Visual Object and Space Perception battery), and visual field tests. We also implemented a face/no face categorization test with Mooney faces during which participants categorized upright or inverted two-toned black-and-white face profiles as a face or not a face (Busigny et al., 2010). Finally, we implemented five tests of face individuation as follows: (1) The classical neuropsychological Benton Face Recognition Test. Subjects were required to match a face among distractor faces varying in viewpoint and lighting (in this test, all faces are shown simultaneously) (Benton et al., 1983). (2) An old/new face recognition test (Busigny et al., 2010). Subjects viewed a series of face 
images during a study phase; and then during a test phase, they were presented with new and old images and were asked to respond whether each image was old or new. (3) The Cambridge Face Memory Test (Duchaine and Nakayama, 2006). Subjects first studied 6 faces. Then, in 72 trials, subjects were asked to identify which of three faces was one that they had previously studied. Throughout the experiment, trials became increasingly harder as faces were shown in new views, illuminations, and with added noise. (4) Face matching across viewpoints (Busigny et al., 2010). Subjects were shown a frontal face on the top of the screen and two three-quarter views of faces below. They were then asked to indicate which of the two three-quarter probes on the bottom were the same as the frontal view on top. (5) Delayed face matching of upright and inverted faces (Busigny et al., 2010).

It should be noted that, when asked specifically about her face recognition abilities a few months before the operation (at the time of the SEEG implantation), Patient S.P. acknowledged that she "had never been very good at recognizing people from their face." We should, however, remain cautious with such statements, given that there is little relationship between subjective reports of face recognition abilities and behavioral measures of face matching and face memory, such as the Cambridge Face Memory Test (Palermo et al., 2016). Five control female participants (age, sex, and education level matched) also participated in two behavioral sessions taken 12 months apart to assess any variations in test-retest performance on these behavioral tests. The modified $t$ test of Crawford-Howell for single-case studies (Crawford and Howell, 1998) was used to compare the results of Patient S.P. and controls. Five typical control female subjects $31-40$ years of age (mean age $35 \pm 4.6$ years) participated in behavioral testing S1. Four of these subjects (mean age $36 \pm 5.5$ years) participated in a second behavioral testing session a year later.

\section{fMRI and DWI in control subjects}

Subjects. Ten adults (19-45 years old; 3 females; all right-handed) were recruited to serve as controls for the selectivity and reliability analyses. All participants were scanned at Stanford University and gave their written informed consent. Procedures were approved by the Stanford Internal Review Board on Human Subjects Research.

Scanning. Subjects were scanned on a 3T GE scanner at the Center for Neurobiological Imaging at Stanford University.

Anatomical brain volumes. A high-resolution anatomical volume of the whole brain was acquired with a T1-weighted BRAVO pulse sequence $\left(\mathrm{TR}=450 \mathrm{~ms}\right.$, flip angle $=12^{\circ}, 1 \mathrm{NEX}, \mathrm{FOV}=240 \mathrm{~mm}$, resolution: 1.0 $\mathrm{mm}$ isotropic).

$f M R I$. Because the comparisons of Patient S.P.'s brain responses were conducted across sessions, all individuals participated in two fMRI scanning sessions. Participants were scanned using a T2 ${ }^{\star}$ sequence $(\mathrm{TE}=30$ $\mathrm{ms}$, TR $=2000 \mathrm{~ms}$, flip angle $=77^{\circ}$, bandwidth $=128 \mathrm{kHz}$, resolution: $2.4 \mathrm{~mm}$ isotropic voxels, 34 slices, $\mathrm{FOV}=192 \mathrm{~mm}$ ).

Functional localizer. Participants viewed a block design experiment consisting of images of faces and nonface categories presented at $1 \mathrm{~Hz}$. The nonface categories were as follows: places (houses/corridors), bodies, limbs, characters (pseudowords/numbers), and objects (cars/guitars). Participants were instructed to respond by button press when a phase-scrambled image appeared (Stigliani et al., 2015). Subjects participated in 2 runs in S1 and 2 runs in S2 using different stimuli.

$f M R I$ data analysis. Data were analyzed with the same pipeline as described for Patient S.P.

ROI definition and mean selectivity measurements. IOG-faces (10 of 10 participants), pFus-faces (10 of 10 participants), pSTS-faces ( 8 of 10 participants), and mSTS-faces (10 of 10 participants) were defined as in Patient S.P. (threshold of $t>3$, voxel level) and in our prior experiments (Weiner et al., 2010; Stigliani et al., 2015) using both anatomical and functional criteria for the mean selectivity analyses. Each ROI was defined in S1, and mean selectivity was calculated for runs 1 and 2 in S2 (Fig. 3E; error bars indicate SDs between runs 1 and 2). It is important to underscore that only the categories overlapping with Patient S.P.'s experiment (faces, limbs, objects, and houses) were used to calculate selectivity.

ROI definition and reliability measurements. Because we only had 4 runs for each control subject ( 2 runs in S1 and 2 runs in S2), and to ensure that all data were independent, we defined ROIs from run 1 in S1 and calculated the reliability of face selectivity between run 2 in $S 1$ and (1) run 1 in S2 and (2) run 2 in S2. This resulted in two measurements per subject. Because ROIs were now defined with only 1 run of data, mSTSfaces were only identifiable in 8 of 10 subjects. Reliability was calculated in the same way as in Patient S.P. as described above.

$D W I$. Because we identify a new pathway connecting V7/IPS-0 to pFus-faces/FFA-1 in Patient S.P., we sought to validate this pathway in control participants. Five (24-45 years old; 2 female; all right-handed) of our typical controls participated in 2 runs of a diffusion-weighted dualspin echo sequence $(60$ slices, $\mathrm{TE}=96.8 \mathrm{~ms}, \mathrm{TR}=8000 \mathrm{~ms}$, 96 diffusion directions, $\mathrm{b}=2000 \mathrm{~s} / \mathrm{mm}^{2}$, voxel size $=2 \times 2 \times 2 \mathrm{~mm}$ ). Ten nondiffusion-weighted images were collected at the beginning of each scan.

DWI data analysis. Preprocessing was done as described above, and MRtrix probabilistic tractography was run using a harmonic envelope of 8 on the first diffusion dataset (as in Gomez et al., 2015). We statistically validated these new fibers connecting V7/IPS-0 and pFus-faces using LiFE (Pestilli et al., 2014). $d^{\prime}$ values $>4$ indicate statistically significant tracts, and the average of these tracts across subjects was $31.52 \pm 4.13$ (SD across subjects; see Fig. 10).

\section{Results}

\section{Before surgery, Patient S.P.'s face network is not significantly} different than control participants

Preoperatively, Patient S.P.'s face-selective regions are located on the IOG (IOG-faces/OFA), lateral FG (pFus-faces/FFA-1 and mFus-faces/FFA-2), as well as the posterior and main branches of the STS (pSTS-faces and, mSTS-faces, respectively; Figs. 3, 4), which is consistent with measurements of the face network in typical subjects (Weiner and Grill-Spector, 2013; Weiner et al., 2014). Independent measurements with event-related fMRI (Fig. $3 B$ ) and iEEG (Fig. 3C) show that the selectivity of these regions is also characteristic, manifesting as higher responses to faces than nonfaces $(p<0.01)$. IEEG recordings in pFus-faces/FFA-1 further showed characteristic latencies of this selectivity emerging at $\sim 150$ ms after stimulus onset (Allison et al., 1994; Parvizi et al., 2012).

To examine the typicality of Patient S.P.'s face network before resection with respect to face selectivity, we compared Patient S.P.'s face-selective regions with those of 10 healthy control participants. Independent analyses of mean selectivity in right hemisphere face-selective regions show that selectivity of Patient S.P.'s face network was not different from the mean selectivity in controls (modified $t$ test of Crawford-Howell for single-case studies: all $t$ values $<1.9$; all $p$ values $>0.10$; Fig. $3 D$ ) or from selectivity in individual subjects (Fig. 3E). Additionally, comparison of the variability in measurements of selectivity across runs was also not different across Patient S.P. and controls in any of the faceselective regions (modified $t$ test of Crawford-Howell for singlecase studies comparing the differences between runs 1 and 2 in Patient S.P. and controls: all $t$ values $<0.72$; all $p$ values $>0.50$; Fig. $3 E$ ). It is important to underscore that, because of independent analyses, selectivity can be below the threshold used to define the voxels of the ROI (Fig. 3E, dotted line). This occurs in both Patient S.P. and controls, indicating that fluctuations of selectivity across runs occur whether the individual is a patient or not. Together, these pre-resection measurements validate that, at least as far as category selectivity is concerned, the cortical layout, response characteristics, selectivity, and run-to-run variability of Patient S.P.'s face network before resection are not significantly different from healthy controls.

\section{Behavioral performance is stable before and after surgery}

Behaviorally, before surgery, Patient S.P. was in the normal range for categorizing two-tone images as either faces or nonfaces 
A

\section{Pre-resection}

S1: 1 month prior
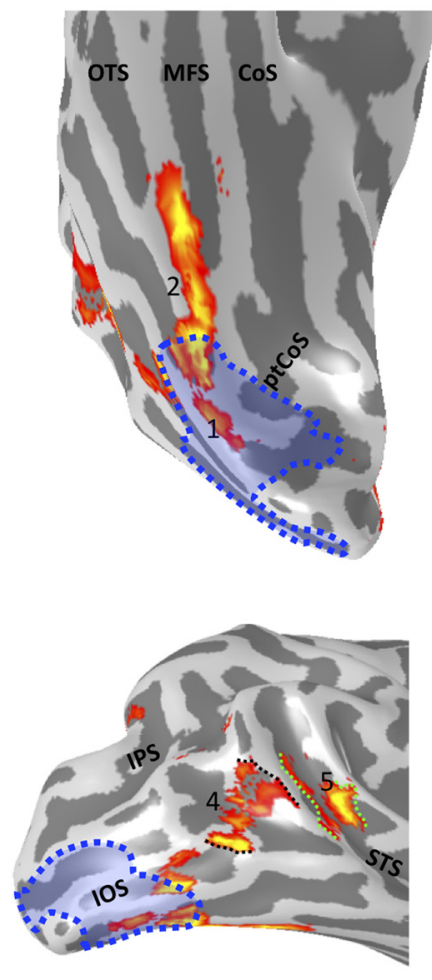

B
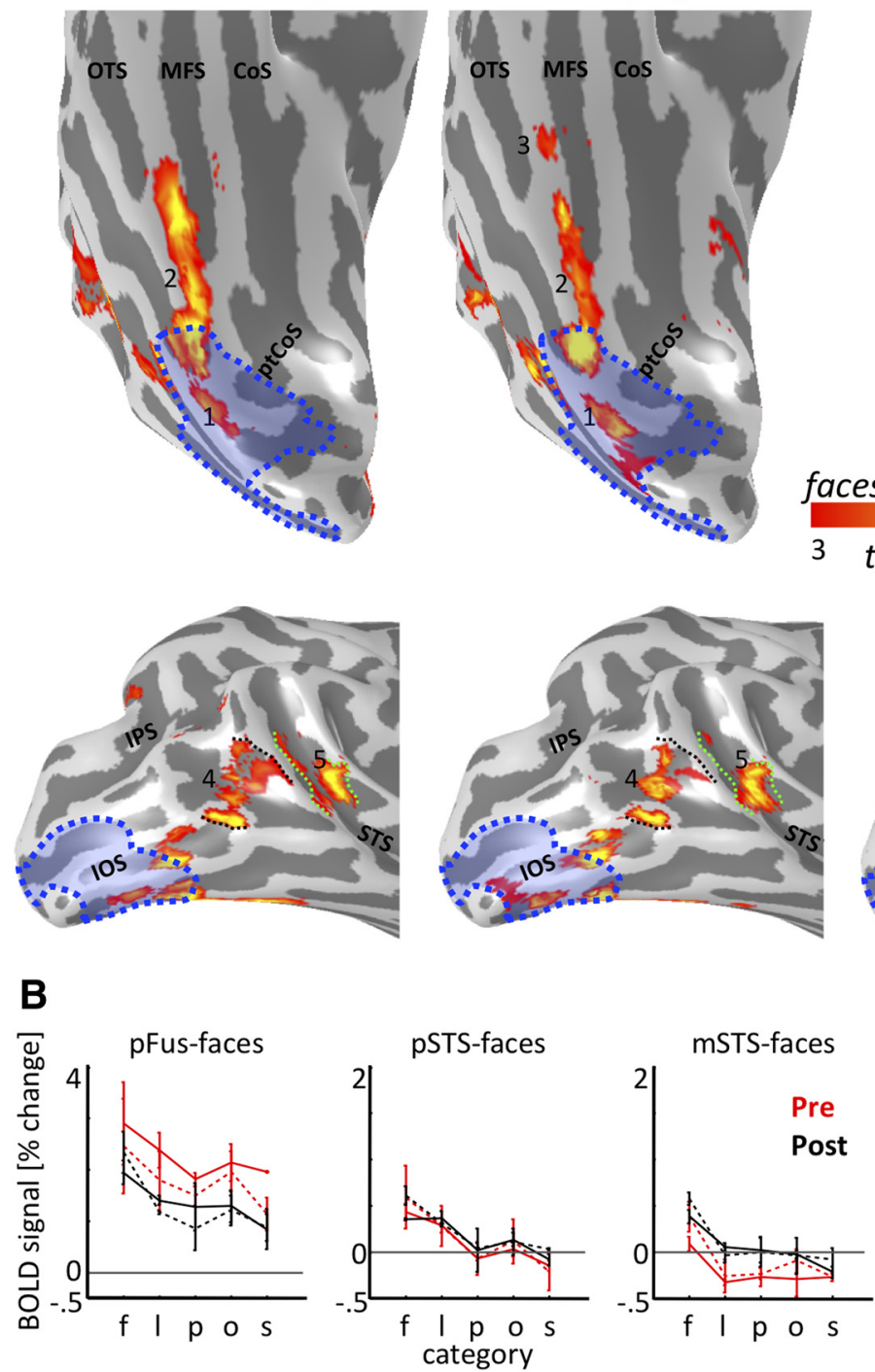

Post-resection

S3: 1 month after
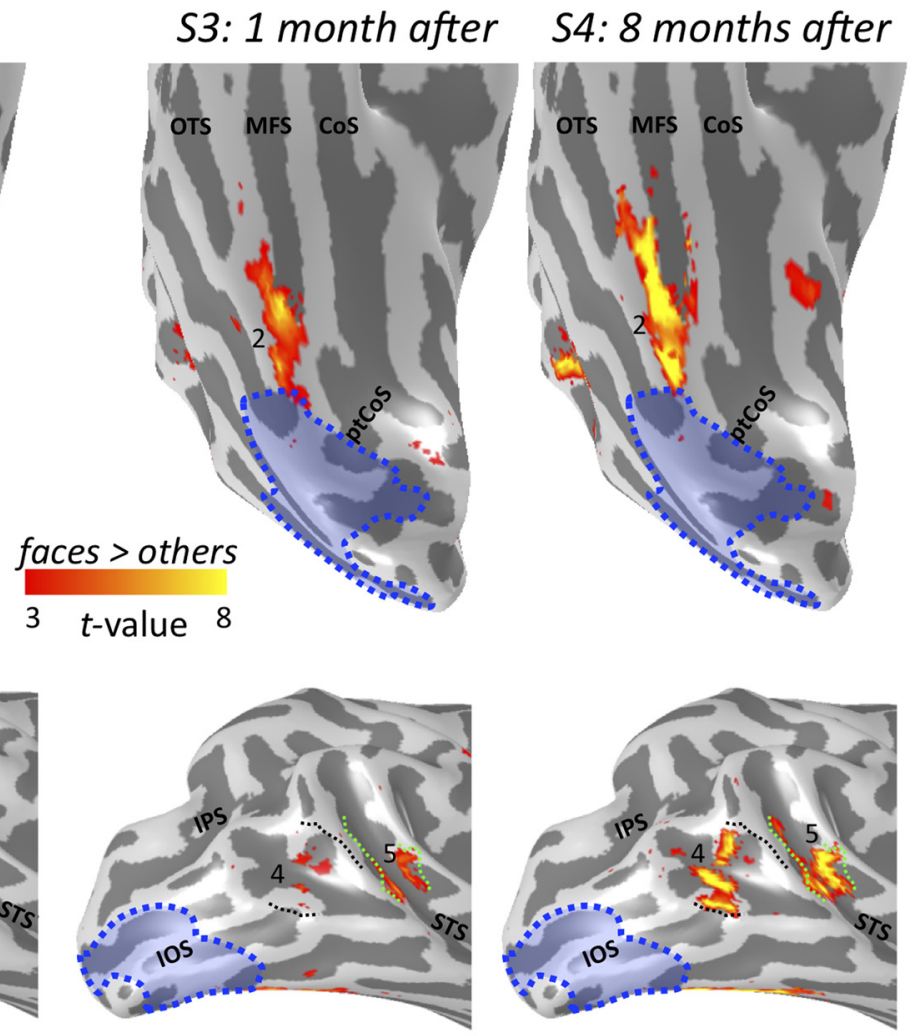

C

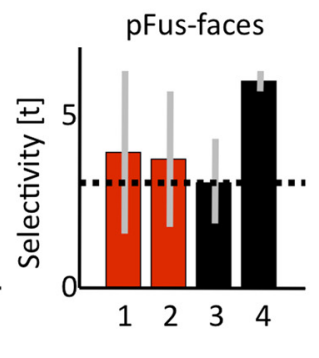

mSTS-faces
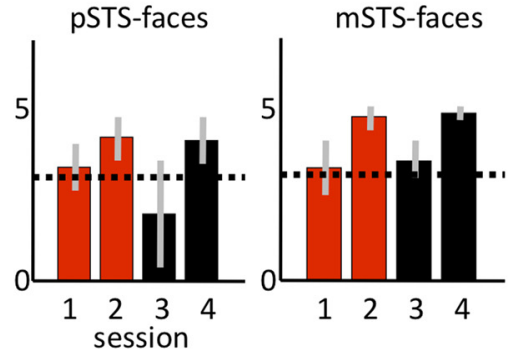

Figure 4. The face network is stable after resection. $A$, The face network before and after resection on the inflated cortical reconstruction of the right hemisphere of Patient $S$.P. before resection. 1, IOG-faces/OFA; 2, pFus-faces/FFA-1; 3, mFus-faces/FFA-2; 4, pSTS-faces; 5, mSTS-faces. IOG-faces/OFA (1) and the posterior portion of pFus-faces/FFA-1 (2) have been resected. Blue shading represents resected cortex. Black represents superior and inferior extent of $\mathrm{pSTS}$-faces in $\mathrm{S1}$. Green represents posterior and anterior extent of mSTS-faces in S1. The unnumbered region on the lateral surface overlapping the IOS was also partially resected, but this region is not typically included in models of the face network because it exhibits comparable selectivity with other animate stimuli (see Materials and Methods). B, Mean fMRI response amplitude (\% change) as a function of category in face-selective regions before (red) and after (black) resection. Error bars indicate SDs across runs in each session. S1, S3, Solid. S2, S4, Dotted. f, Faces; I, limbs; p, places; 0, objects; S, scrambled. Conditions are ordered by rank. C, Face selectivity as a function of session. Error bars indicate mean selectivity across voxels of independently defined ROIs for run 1 and run 2 in each session. Error bars indicate SDs across runs in each session. Dotted line indicates the statistical threshold $(t>3)$ used to define each region from independent data. CoS, Collateral sulcus; IOS, inferior occipital sulcus; IPS, intraparietal sulcus; MFS, mid-fusiform sulcus; 0TS, occipitotemporal sulcus; ptCoS, posterior transverse collateral sulcus.

(Mooney faces, Patient S.P.: 86.3\%; age- and gender-matched controls: $90.3 \pm 5 \%$, no significant difference, $t=0.677, p=$ 0.54). Patient S.P. was also in the normal range on the old/new face recognition test (Patient S.P.: 90\%; controls: $94 \pm 3 \%, t=$ $1.299, p=0.132)$. Although she performed well above chance level, she was impaired relative to controls on behavioral tasks requiring unfamiliar face individuation (Table 1). However, as illustrated in case studies in patients with long-term brain damage (Schiltz et al., 2006; Steeves et al., 2009), behavioral impairments in face individuation do not impede the ability to measure face-selective responses (i.e., faces $>$ nonfaces) in high-level visual cortex. Thus, before surgery, Patient S.P. displayed an intact face network and abilities within the normal range for both remembering faces and categorizing faces from other stimuli.

After resection, Patient S.P. remained in the normal performance range for categorizing two-tone images as either faces or nonfaces (Mooney faces, Patient S.P.: 84.4\%, no significant difference from age- and gender-matched controls: $92.8 \pm 5 \%, t=$ $1.439, p=0.123$; Table 1). It is important to highlight that this is a difficult task and subjects cannot rely on local, low-level features to perform well. For example, a visual agnosic patient, such as Patient D.F., is at chance at this task, even when she can discern a face from an object in other formats (Steeves et al., 2006). Finally, Patient S.P. was not our worst subject, as one of our typical con- 
Table 1. Behavioral performance of Patient S.P. and age- and gender-matched control participants

\begin{tabular}{|c|c|c|c|c|}
\hline & Before operation & $\begin{array}{l}\text { After operation } \\
\text { (1 month) }\end{array}$ & Control participants & $\begin{array}{l}\text { Control participants } \\
\text { (1 year after) }\end{array}$ \\
\hline Categorize upright/inverted Mooney faces & $86.3 \%$ & $84.4 \%$ & $90.3 \%( \pm 5.4)$ & $92.8 \%( \pm 5.2)$ \\
\hline Old/new face memory test & $90 \%$ & $93.3 \%$ & $94 \%( \pm 2.8)$ & $95 \%( \pm 4.3)$ \\
\hline Cambridge Face Memory Test & $41 / 72^{* *}$ & $40 / 72^{* *}$ & $59.2 / 72( \pm 8.6)$ & $59.2 / 72( \pm 10.4)$ \\
\hline Face matching across viewpoints & $65 \% *$ & $64 \% *$ & $82.2 \%( \pm 7.0)$ & $87 \%( \pm 6.2)$ \\
\hline \multicolumn{5}{|l|}{ Face matching } \\
\hline Age (yr) & 36 & 36 & $35 \pm 5$ & $36 \pm 5$ \\
\hline
\end{tabular}

*Impaired scores compared with normal controls ( $p<0.05)$.

**Impaired scores according to Benton Face Recognition Test and Cambridge Face Memory Test scales.

trols scored less than Patient S.P. in this task. Given the present tests before and after resection, we believe an accurate interpretation of these results is that Patient S.P.'s ability to categorize a face as a face is intact.

Additionally, Patient S.P.'s IQ remained in the normal range (score: 101), and her score at all 8 tests of the Visual Object and Space Perception battery remained at ceiling. Patient S.P.'s ability to individuate faces was still below controls in several tasks but did not worsen following surgery, and her performance on the old/new face recognition memory task remained in the typical range (Patient S.P.: 93.3\%; controls: $95 \pm 4 \%$, no significant difference, $t=0.353, p=0.374$; Table 1 ). Together, Patient S.P.'s behavioral performance in all of the face tasks was stable after compared with before surgery despite having a left hemianopic central scotoma after surgery.

The cortical layout of the face network is stable after resection of IOG-faces/OFA and the posterior portion of pFus-faces/FFA-1

The resection included the right IOG and portions of surrounding gyri, sulci, and white matter (Figs. 1, 3, 4). We aligned data from all fMRI sessions to the presurgical anatomy after defining the locus of the resected tissue (Figs. 1, 4). This approach revealed that all of IOG-faces and the posterior $23.3 \%$ of pFus-faces were surgically removed.

Surprisingly, after resection, the cortical topology and extent of pFus-faces in ventral temporal cortex (VTC) remained stable. Even the posterior portion of pFus-faces, which abutted the resection, was similar to its presurgery location. Regions along the STS displayed more variability after resection than regions in VTC (Fig. 4A, bottom). For example, 1 month after surgery, pSTS-faces and mSTS-faces were less activated than before surgery. However, 8 months after surgery, both regions were activated to a similar extent before surgery.
In contrast to the general stability of pFus-faces, pSTS-faces, and mSTS-faces in the resected hemisphere, we observed instabilities in the localization of mFus-faces/FFA-2 before resection and in a region on the IOS after resection. The instability of mFus-faces across sessions is likely driven by low tSNR from the ear canal artifact and not by the surgery, as the tSNR in mFusfaces before resection was lower than the minimum for reliable measurements (see Materials and Methods). Before resection, the IOS responded similarly to faces and bodies (no significant difference, $p=0.60$ ) and was significantly modulated by visual stimuli (variance of the time-series explained by the GLM is $59.1 \pm$ $8.9 \%$ ). The surgery resected $26.9 \%$ of the IOS. Consequently, the variance of the IOS time-series explained by the GLM dropped close to zero $(5.4 \pm 2.0 \%)$ after resection, which was significantly $(p<0.008)$ lower than before surgery. This indicates that, after the resection, this region is not significantly modulated by visual stimuli modeled by the GLM. However, because the IOS is not included in traditional neurocognitive models of face perception and is also not strictly face-selective in either Patient S.P. or controls (see Materials and Methods), we focus the remainder of our analyses on pFus-faces, pSTS-faces, and mSTS-faces.

In addition to qualitatively examining the cortical topology of right pFus-faces, pSTS-faces, and mSTS-faces, we also quantitatively compared the mean response amplitudes and face selectivity within each region before and after resection. These analyses revealed that the resection differentially affected the mean BOLD response across face-selective regions. In pFus-faces, overall response amplitudes were lower after than before resection, whereas in mSTS-faces, the opposite was true; meanwhile in pSTS-faces, there was no substantial change in the overall signal level (Fig. 4B). Indeed, a three-way ANOVA with factors region (right pFus-faces/mSTS-faces/pSTS-faces), category, and resection (before resection/after resection) yielded a region $\times$ resection interaction $\left(F_{(2,90)}=34.3, p<10^{-3}\right)$. Importantly, the

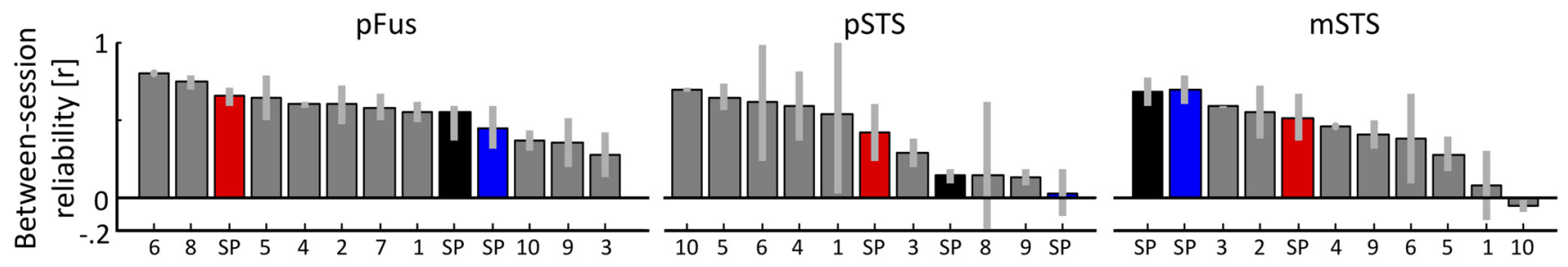

Figure 5. The reliability of distributed patterns of face selectivity in Patient S.P. is not significantly different than controls. Each bar represents data from a single subject and reflects the correlation between the distributed face selectivity in individual voxels across runs from two different sessions. Gray represents controls. Red represents Patient S.P. before resection. Black represents Patient S.P. after resection. Blue represents correlation between pre-resection and post-resection distributed face selectivity in Patient S.P. The $X$-axis is ranked in a descending order to indicate where Patient S.P. falls in the distribution of typical participants. 
A
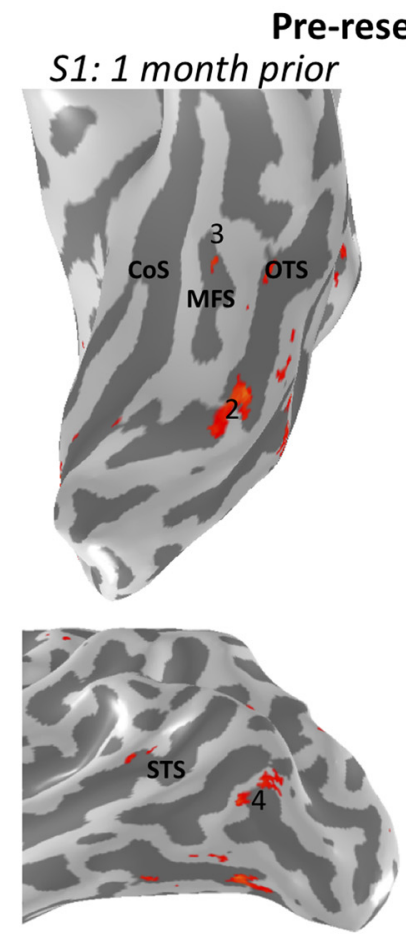

B

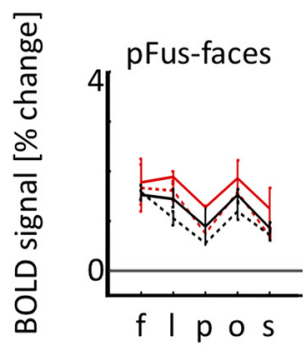

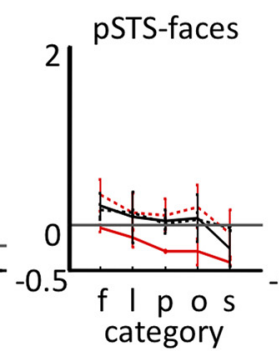

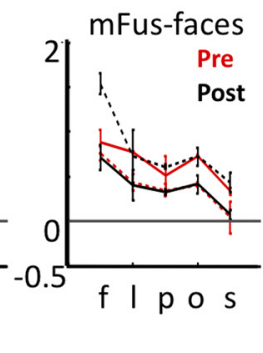

S2: 4 days prior
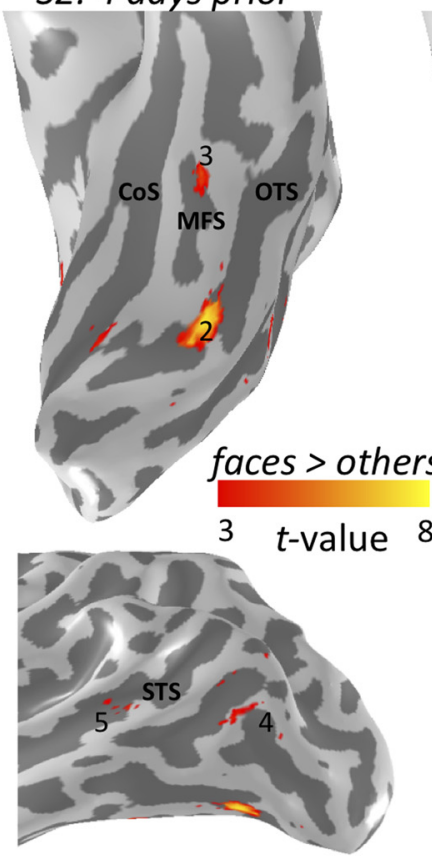

S3: 1 month after

C

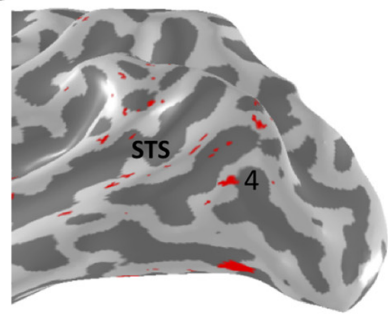

Post-resection
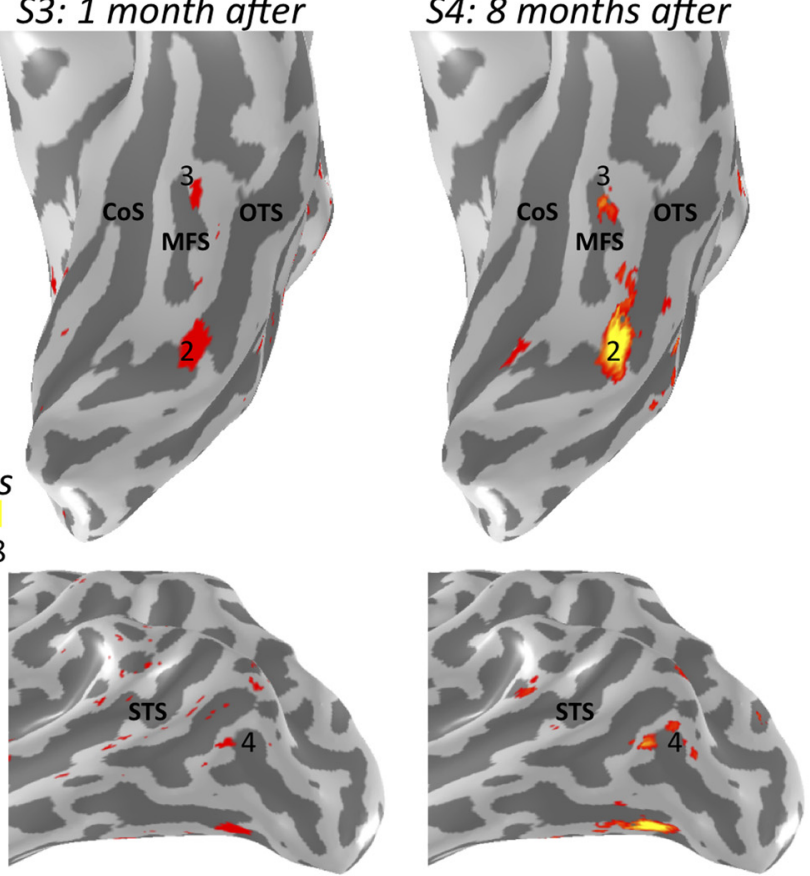

Figure 6. Weak face selectivity in the left hemisphere of Patient S.P. A, Face-selective network in VTC (top) and LOTC (bottom) before resection and after resection projected to the inflated cortical reconstruction of the left hemisphere of Patient S.P. 2, pFus-faces/FFA-1; 3, mFus-faces/FFA-2; 4, pSTS-faces; 5, mSTS-faces. Left IOG-faces/OFA (1) could not be identified in any session, whereas left mSTS-faces (5) could only be identified in S2. COS, Collateral sulcus; MFS, mid-fusiform sulcus; 0TS, occipitotemporal sulcus. $\boldsymbol{B}, \boldsymbol{C}$, Same layout as Figure 4, but for face-selective regions in the left hemisphere. Red represents before surgery. Black represents after surgery. S1, S3, Solid. S2, S4, Dotted. f, Faces; I, limbs; p, places; 0, objects; S, scrambled. Regions in the left hemisphere have lower face selectivity than the right hemisphere (Fig. 4).

resection only affected the overall magnitude of the signal but did not change the profile of response within each region (no region $\times$ category $\times$ resection interaction: $\left.F_{(8,90)}=0.27, p=0.98\right)$. Specifically, each ROI showed the same ranking of responses across categories of stimuli before compared with after resection.

Likewise, we find that all face-selective regions maintain significant face selectivity after the resection despite fluctuations in the magnitude of selectivity (Fig. 4C). A two-way ANOVA with factors region (right pFus-faces/pSTS-faces/mSTS-faces) and session (1/2/ $3 / 4)$ revealed a main effect of session $\left(F_{(3,12)}=3.77, p<0.04\right)$. However, there were no significant differences in the level of selectivity after compared with before the resection (two-way ANOVA with factors of region (pFus-faces/pSTS-faces/mSTS-faces) and resection (before/after), no effect of resection, $\left.F_{(1,18)}=.01, p=0.9\right)$. Importantly, after resection, both the mean selectivity (all $t$ values $<1.96$; all $p$ values $>0.08$ ) of each face-selective region in Patient S.P. and the fluctuations in selectivity between runs within each session (all $t$ values $<1.18$; all $p$ values $>0.27$ ) were not different than controls. These results indicate that, although there are fluctuations in the selectivity value across sessions (Fig. 4C), these fluctuations are comparable before and after resection, are comparable with controls (Fig. 3E), and are not due to a general increase or decrease in selectivity after surgery.

The present analyses underscore the importance of acquiring multiple functional measurements before and after resection. For example, in pSTS-faces and mSTS-faces, S1 (pre) and S3 (post) have comparable selectivity values, as do S2 (pre) and S4 (post; Fig. 4C). However, if we had only acquired a single session before and a single session after surgery, we would have arrived at different conclusions based on the particular combination of the subset of sessions we may have obtained. For instance, had we only acquired S1 and S4, we would have concluded that the resection increases selectivity. In contrast, had we only acquired S2 and S3, we would have arrived at an opposite conclusion: that the resection decreases selectivity. However, both of these conclusions are inaccurate. Instead, our data show that there are fluctuations in selectivity across sessions regardless of the resection. 
A

pSTS-faces

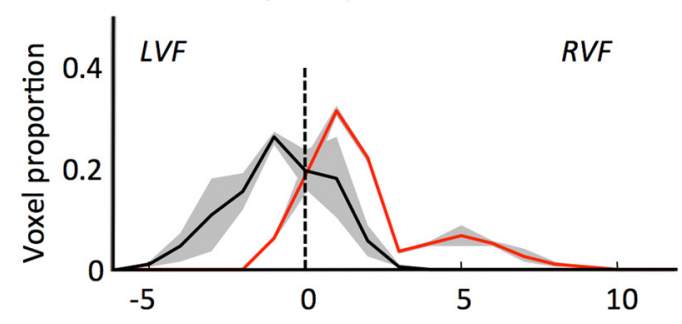

C

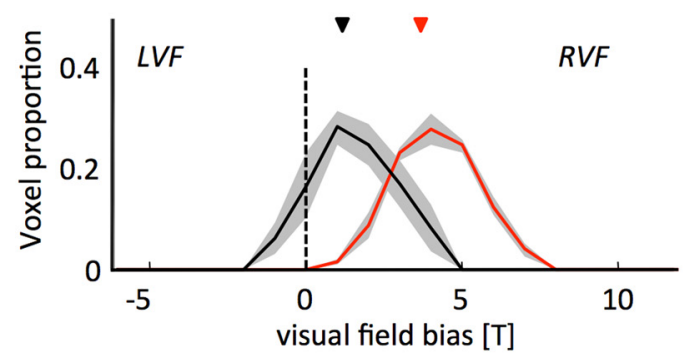

B
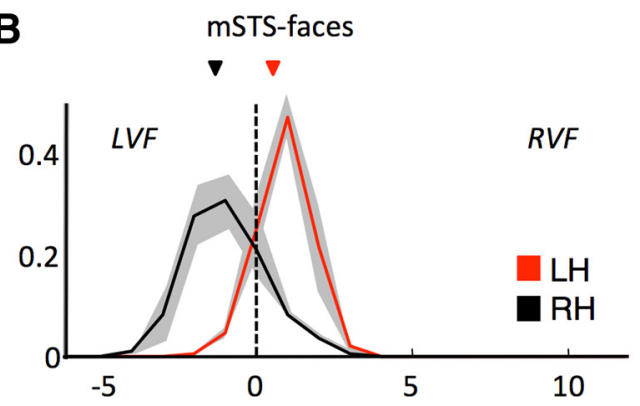

D

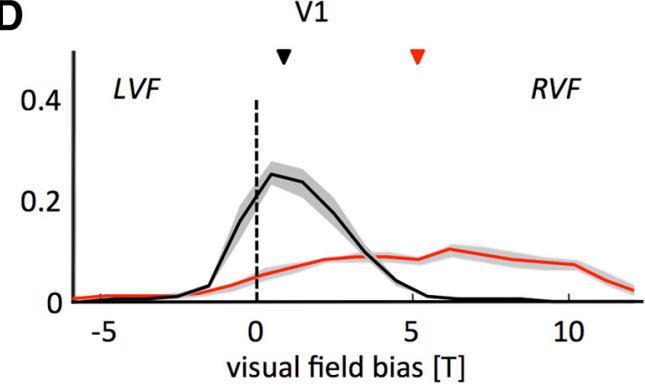

Figure 7. Typical contralateral bias in the superior temporal sulcus, but atypical ipsilateral bias in the fusiform gyrus and V1, after removal of IOG-faces/OFA. Histograms represent the proportion of voxels biased to the right visual field (positive $t$ value) or the left visual field (negative $t$ value) for functional ROls in the right (black) and left (red) hemispheres. pSTS-faces and mSTS-faces in the resected hemisphere have the typical contralateral bias displayed by visual areas, whereas pFus-faces and V1 in the resected hemisphere have an unexpected ipsilateral bias. $\boldsymbol{A}$, pSTS-faces. $\boldsymbol{B}$, mSTS-faces. C, pFus-faces. D, V1. LH, Left hemisphere; LVF, left visual field; RH, right hemisphere; RVF, right visual field. Light gray shading represents SE across voxels.

The resection differentially affects the distributed pattern of face selectivity within regions of the face network surviving the resection

To complement our mean signal and mean selectivity analyses and to further quantify the stable and plastic features of pFusfaces, pSTS-faces, and mSTS-faces, we calculated the betweensession reliability of distributed face selectivity across voxels within each face-selective region (see Materials and Methods). Before resection, the distributed patterns of face selectivity within right pFus $(r=0.65 \pm 0.06)$, pSTS $(r=0.42 \pm 0.19)$, and mSTS $(r=0.51 \pm 0.15)$ were positively correlated between each pair of experimental runs taken between pre-resection sessions (all $p$ values $<10^{-4}$; Fig. 5, red). After resection, the reliability of distributed face selectivity across voxels was also positively correlated across experimental runs between sessions in pFus $(r=$ $0.45 \pm 0.11, p<0.04)$ and $\operatorname{mSTS}(r=0.70 \pm 0.09, p<0.006)$, but not in pSTS ( $r=0.14 \pm 0.05, p=0.08$; Fig. 5 , black). A two-way ANOVA with region and resection as factors revealed a main effect of region $\left(F_{(2,18)}=17.1, p<10^{-4}\right)$, a borderline effect of resection $\left(F_{(1,18)}=4.2, p=0.06\right)$, and a significant region $\times$ resection interaction $\left(F_{(2,18)}=8.6, p<0.002\right)$. Thus, the resection differentially affected the distributed pattern of face selectivity within regions of the face network surviving the surgery.

While these analyses separately assess the stability of distributed face selectivity patterns before versus after resection, they do not directly quantify the relationship between face selectivity patterns before resection to after resection. To do so, we calculated the correlation among distributed patterns of face selectivity before resection compared with after resection. This analysis revealed two main findings. First, the distributed patterns of face selectivity were positively correlated between pre-resection and post-resection sessions in pFus $(r=0.48 \pm 0.14)$ and $\operatorname{mSTS}(r=$ $0.68 \pm 0.10$; all $p$ values $<10^{-4}$; Fig. 5 , blue). In contrast, only 5 of 16 pre-resection and post-resection pairs had a positively correlated relationship in pSTS, and the average $(r=0.03 \pm 0.15)$ was not significantly different from zero $\left(t_{(15)}=0.2, p=0.85\right)$. This decorrelated relationship can be interpreted as a change in the distributed pattern of face selectivity across voxels within pSTSfaces preoperatively and postoperatively. Second, the correlations between distributed face selectivity patterns pre-resection and post-resection sessions in Patient S.P. (Fig. 5, blue) were not significantly different from controls (Fig. 5, light gray) in pFus (all $t$ values $<0.57$, all $p$ values $>0.58$ ), and mSTS (all $t$ values $<1.5$; all $p$ values $>0.17$ ). Even in pSTS, where we find a decorrelated relationship in distributed face selectivity between preresection and post-resection experimental runs, this difference was not significant compared with controls, as two of our controls also had nonreproducible distributed face selectivity across sessions (all $t$ values $<1.7$, all $p$ values $>0.13$ ).

These findings emphasize the value of pre-resection and postresection assessments within the same patient in combination with benchmarks relative to controls. For example, if we only had post-resection measurements in Patient S.P., as is common in cases with patients that have long-term brain damage, we would have concluded that the reliability of face selectivity within pSTSfaces is within the range of controls. Although that is accurate, the incorporation of pre-resection measurements enable us to conclude that the distributed pattern of face selectivity in Patient S.P. is different after compared with before surgery within pSTSfaces, but not in other regions of the face network. These conclusions would not be possible without (1) multiple pre-resection and post-resection measurements in Patient S.P., (2) multiple scales of analysis (e.g., analyses of mean selectivity as well as distributed patterns across voxels), and (3) analogous measurements in multiple sessions in typical controls.

Examining the role of the left hemisphere in the post-resection stability of the face network

Our neuroimaging data indicate that the cortical layout and selectivity of the right hemispheric face network remained largely 


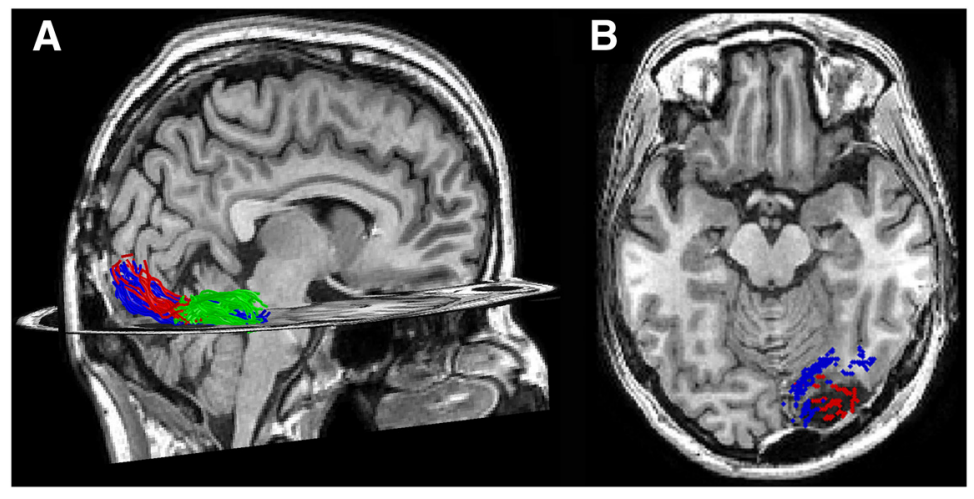

Figure 8. White matter tracts connecting early visual cortex to IOG-faces, but not pFus-faces, were likely resected. A, Preresection measurement of white matter tracts connecting the central $5^{\circ}$ of V1/V2 and pFus-faces/FFA-1 (blue) and IOG-faces/OFA (red), respectively, projected to the post-resection T1. White matter tracts connecting IOG-faces/OFA and pFus-faces/FFA-1 (green) are also depicted. $\boldsymbol{B}$, Axial slice illustrating endpoints of fascicles from the central $5^{\circ}$ of V1/V2 to pFus-faces/FFA-1 (blue) and IOG-faces/OFA (red) projected onto the post-resection T1. The former largely run a more medial route, whereas the latter terminate directly into the resection.

A

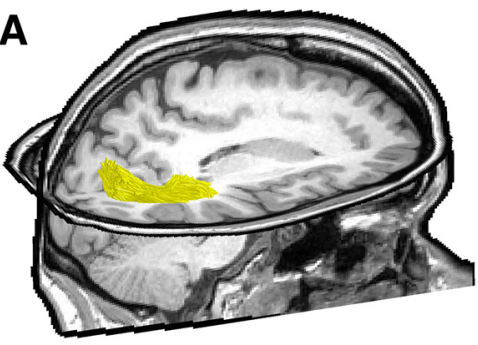

B

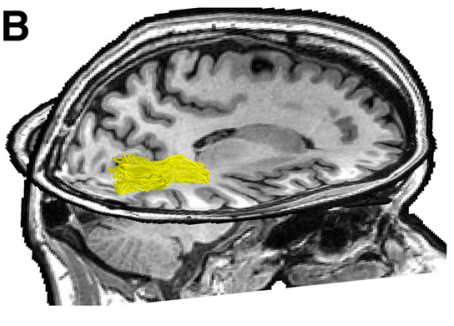

Figure 9. White matter fascicles extending from the periphery of early visual cortex likely contribute to the stability of STS face-selective regions postoperatively. White matter tracts (gold) connecting the far periphery $\left(>20^{\circ}\right)$ of V1/V2 and pSTS and mSTS face-selective regions, respectively, in the resected hemisphere. $\boldsymbol{A}$, Before surgery. $\boldsymbol{B}$, After surgery. To display the tracts clearly, functional regions are not shown.

stable following removal of the right IOG. Although this finding is consistent with predictions from a multiple route network with nonhierarchical components, it is also possible that this resiliency may be achieved via inputs from face-selective regions in the intact, left hemisphere. However, several pieces of evidence suggest that the resiliency of the right hemispheric face network is not driven by inputs from the left face network. First, it is unlikely that inputs from left IOG-faces support the stability of right pFus-faces because we were unable to identify IOG-faces in the left hemisphere in any session (Fig. 6A) and left IOG-faces is also not always detected in typical individuals (Zhen et al., 2015). Second, face selectivity in the left hemisphere (Fig. 6C) is consistently weaker than in the right hemisphere both before and after resection (three-way ANOVA with factors region, hemisphere, and resection yielded a main effect of hemisphere: $F_{(1,24)}=19.9$, $p<10^{-3}$, and no interactions: all $F$ values $<1.8$; all $p$ values $>0.19)$. These findings are consistent with the literature showing a larger spatial extent, selectivity, and response amplitude to faces in the right compared with the left hemisphere (Sergent et al., 1992; Kanwisher et al., 1997; Rossion et al., 2012; Zhen et al., 2015).

To directly examine the role of the left hemisphere, we conducted an experiment in which the position of visual images from different categories was presented at the center, right, or left of fixation (see Materials and Methods). A characteristic feature of visual areas, including high-level visual regions (Hemond et al., 2007; Kay et al., 2015), is a contralateral preference for visual stimuli: that is, stronger neural responses to stimuli presented in the right visual field compared with stimuli presented in the left visual field in the left hemisphere and vice versa in the right hemisphere; this experiment directly tests the role of the left hemisphere in supporting the stability of the right hemispheric face-selective regions after resection. Based on prior evidence (Felleman and Van Essen, 1991), a contralateral preference in face-selective regions within the resected hemisphere likely reflects signals propagated through within-hemisphere connections. On the contrary, an ipsilateral preference would suggest that inputs from the left hemisphere affect responses in the resected hemisphere.

As expected, in the unresected, left hemisphere, face-selective regions showed a contralateral preference for stimuli in the right visual field. In the resected, right hemisphere, pSTS-faces and mSTS-faces also showed the characteristic contralateral preference for stimuli in the left visual field (Fig. $7 A, B$ ). However, even though the visual field preference in right pFusfaces is significantly different from left pFus-faces $\left(p<10^{-3}\right)$, it exhibited an ipsilateral, not a contralateral, preference (Fig. 7C). Although this suggests that inputs from the left hemisphere contribute to the stability of right pFus-faces, this reduced contralateral preference is already present in the central $5^{\circ}$ of right V1 (Fig. $7 D$ ). Thus, the ipsilateral bias in pFusfaces in the right hemisphere likely originates in the central $5^{\circ}$ of $\mathrm{V} 1$ of the right hemisphere (consistent with the postoperative central hemianopic scotoma), which then communicates signals downstream to right pFus-faces.

Together, these results show that: (1) the resiliency of the right hemispheric face network is likely not driven by inputs from the left face network; (2) the left hemisphere may play a role for the stability of right pFus-faces, but these signals may be communicated directly from right $\mathrm{V} 1$ to downstream right pFus-faces; and (3) the contributions of the left hemisphere cannot explain the entire resiliency of the face network because the face-selective regions of the STS maintain a contralateral bias after resection.

\section{Longitudinal and vertical white matter tracts provide an} anatomical infrastructure connecting early visual areas to FG and STS face-selective regions independent of IOG-faces/OFA If the post-resection resiliency of the right hemisphere is largely not driven by inputs from the left hemisphere, are there white matter connections in the right hemisphere that enable signals to reach downstream face-selective regions? Prior studies have identified white matter tracts connecting early visual areas to downstream faceselective regions. For example, Gschwind et al. (2012) have identified separate longitudinal tracts from the calcarine sulcus to faceselective regions on the FG and STS, respectively. Additionally, Kim et al. (2006) have identified vertical tracts connecting dorsal retinotopic areas to face-selective regions on the FG. Thus, it seems plausible that these white matter tracts from outside the face network could provide an anatomical infrastructure supporting both the stable (e.g., face selectivity) and plastic (e.g., representation of visual 
space in right $\mathrm{pFus-faces)} \mathrm{features} \mathrm{of} \mathrm{the} \mathrm{face}$ network following resection of IOG-faces/ OFA. To test this hypothesis, we used DWI and tractography in Patient S.P. before and after resection (see Materials and Methods). DWI after resection enables testing whether these tracts are preserved postoperatively.

Supporting this hypothesis of bypass routes, we find evidence for both longitudinal and vertical tracts connecting early visual areas to downstream face-selective regions independent of IOG-faces. Before resection, we identified separate longitudinal white matter tracts connecting the central $5^{\circ}$ of V1/V2 to IOG-faces/OFA (Fig. $8 A$, red) and to pFus-faces-FFA-1 (Fig. $8 A$, blue) in Patient S.P., which are consistent with prior research in typical subjects (Gschwind et al., 2012; Pyles et al., 2013). However, after resection, we could not measure these ventral longitudinal tracts in Patient S.P. due to diffusivity artifacts produced by the resection (see Materials and Methods). Nevertheless, to gain insight into what pathways might exist after resection, we projected the cortical endpoints of these pre-resection white matter tracts to the post-resection anatomy. This approach reveals that the tracts between early visual cortex and IOG-faces/OFA (red) terminate in the resected tissue and are likely to have been included in the resection. Comparatively, a majority of the tracts from early visual cortex to pFus-faces/FFA-1 (blue) terminate medially in the posterior FG largely outside the resected tissue (Fig. $8 B$ ). Thus, it is likely that these tracts exist after resection and contribute to the resiliency of pFus-faces/FFA-1 after resection.

To test whether there are white matter connections from early visual cortex to STS regions, we seeded an ROI in the far periphery $\left(>20^{\circ}\right)$ of V1/V2, which is outside the scotoma, and examined whether there are connections to STS regions. This analysis identified a set of longitudinal white matter tracts connecting the periphery of early visual cortex and STS face-selective regions both before and after resection (Fig. 9). These tracts may contribute to the preserved contralateral bias in STS regions after resection, as contralateral information originating in the periphery of early visual areas may be communicated through these preserved tracts to STS regions. Identifying white matter tracts connecting the periphery of V1/V2 to STS regions is consistent with evidence of anatomical connections from the periphery of V1/V2 to the periphery of MT in nonhuman primates (Desimone and Ungerleider, 1986). These connections have been postulated to convey signals about dynamic facial motion to the pSTS (O'Toole et al., 2002; Pitcher et al., 2014; Bernstein and Yovel, 2015), as pSTS abuts the peripheral representation of MT in humans (Amano et al., 2009; Weiner and Grill-Spector, 2013).

It is also possible that additional pathways within the right hemisphere provide inputs to STS face-selective regions via MT. These tracts may include within-hemisphere connections from mid-brain structures to MT bypassing V1, such as white matter tracts connecting the lateral geniculate nucleus (Ajina et al., 2015) and the superior colliculus (Ajina et al., 2015) to MT, as well as tracts connecting the pulvinar and MT (Warner et al., 2010). However, due to the artifacts in the postsurgical diffusion scan, we were unable to measure these tracts in $\mathrm{Pa}$ tient S.P.'s brain.

In addition to longitudinal tracts, we were also able to identify vertical tracts connecting dorsal retinotopic areas to ven- tral face-selective regions. Specifically, the VOF is a recently rediscovered major white matter pathway connecting dorsal and ventral components of the occipital lobe (Yeatman et al., 2014; Takemura et al., 2016; Weiner et al., 2016). We first identified the VOF in Patient S.P. and controls (Fig. 10; see Materials and Methods). Importantly, a subcomponent of the VOF connects V7/IPS-0 dorsally to pFus-faces/FFA-1 ventrally in Patient S.P. and controls (Fig. 10, cyan). This subcomponent of the VOF is identifiable in Patient S.P. both before resection (Fig. 10, cyan) and after resection (Fig. 10, purple).

These longitudinal and vertical tracts that are intact in Patient S.P. after surgery provide an anatomical infrastructure for signals to reach downstream face-selective regions on the FG and STS independent of IOG-faces/OFA. Although these tracts provide alternate routes of information flow to downstream face-selective regions, the face network may not always use bypass routes for normative function, which is a hypothesis that can be tested in future research.

\section{Discussion}

We examined the structure and function of the face network in a rare patient before and after removal of right IOG-faces/OFA, which is considered the critical input of the face network according to prevailing models of neural face processing. We found that downstream face-selective regions surviving the resection are largely resilient after surgery, which is consistent with proposals of a multiple route face network with nonhierarchical components (Rossion, 2008; Atkinson and Adolphs, 2011; Pitcher et al., 2011a; Duchaine and Yovel, 2015).

\section{Non strict hierarchical components of the face network enable efficiency and resiliency}

Our results indicate that face-selective neural responses in downstream regions are preserved in patient Patient S.P. as early as 1 month following the removal of right IOG-faces/OFA. These results specify that, even though regions within the face network are functionally (Fairhall and Ishai, 2007; Turk-Browne et al., 2010; Zhu et al., 2011; Lohse et al., 2016) and structurally interconnected (Fairhall and Ishai, 2007; Turk-Browne et al., 2010; Zhu et al., 2011; Gschwind et al., 2012; Pyles et al., 2013; Tavor et al., 2014), the network itself is not arranged in a strict hierarchy where information is always systematically transmitted in a serial manner from IOG-faces/OFA to subsequent processing stages. 
These findings serve as causal evidence that a multiple route network with nonhierarchical components supports face processing (Rossion, 2008; Atkinson and Adolphs, 2011; Pitcher et al., 2011a; Duchaine and Yovel, 2015). Additionally, our DWI measurements show that there are multiple white matter connections from retinotopic regions to the face network (Figs. 8-10), which not only validate recent models (Rossion, 2008; Atkinson and Adolphs, 2011; Pitcher et al., 2011a; Duchaine and Yovel, 2015), but also provide evidence explaining how within hemisphere connections may provide inputs to downstream face-selective regions in the absence of IOG-faces/OFA.

We propose that these additional routes may serve two functions. First, they may increase the efficiency of typically functioning networks by allowing nonserial processing. For example, they may serve as reentrant connections enabling coarse-to-fine processing (Rossion et al., 2003; Rossion, 2008; Goffaux et al., 2011; Jiang et al., 2011), reverse hierarchical processing (Mumford, 1992; Bullier, 2001; Ahissar and Hochstein, 2004), or recurrent processing (Kravitz et al., 2013). Second, they may provide resiliency to the face network by preserving global network functionality following focal network damage. While these white matter tracts verify multiple routes of information from retinotopic areas to downstream face-selective regions, future studies are necessary to clarify their role for normative face processing in typical participants. For example, longitudinal connections may serve to segregate dynamic and static face processing in the lateral and ventral streams, respectively, whereas vertical connections may serve to integrate these two types of information. Further, our findings do not imply that face processing is always nonserial or that there are no hierarchical components in the face network (Kay et al., 2015). Future studies in typical subjects and patients will shed further light on functional processes that are hierarchical and those that are not.

\section{Methodological advancements: the necessity for multiple sessions and metrics}

A unique aspect of our study is that we acquired multiple functional measurements before and after focal resection of human visual cortex. The importance of this approach is that it allows distinguishing meaningful functional changes due to reorganization after surgery from changes that occur due to session-to-session variability. Because pre-resection and post-resection measurements quantify changes between sessions, it is important for future cases to quantify the stability of functional measurements (1) with multiple metrics (mean and voxel level), (2) relative to typical subjects, and (3) across sessions. This suggested approach is not only relevant for cases involving cortical resection or lobectomies, but also any studies that track brain function across an intervention (Op de Beeck et al., 2006) or longitudinal development (Golarai et al., 2007; Scherf et al., 2007) as the session to session variability may outweigh the manipulation itself.

\section{Multiple factors likely contribute to cortical resiliency}

Cortical systems illustrate both stable and plastic features following damage or surgery (Wandell and Smirnakis, 2009). If and how cortex recovers from damage or surgery is linked to the extent of the damage itself. Indeed, research in nonhuman primates reveals that neural responses in inferotemporal cortex can be stable following focal resection but detrimentally affected when extensive amounts of early and intermediate ventral visual areas are removed (Bertini et al., 2004; Buffalo et al., 2005; Kravitz et al., 2013). As such, the extent of the damage directly influences resiliency (or lack thereof) of cortical networks, as well as behav- ior. For example, it may seem surprising that removal of IOGfaces/OFA and the posterior portion of pFus-faces/FFA-1 did not further impair Patient S.P.'s behavioral performance on face individualization tasks as predicted by prior research in humans (Rossion et al., 2003; Bouvier and Engel, 2006; Pitcher et al., 2007; Jonas et al., 2012). However, the present results of stability are less surprising in the context of prior studies in nonhuman primates showing small performance decrements, after (1) removing an entire face patch (little to no impairment) (Heywood and Cowey, 1992), (2) focally deactivating face patches (2\%-5\%) (Afraz et al., 2015), or (3) removing entire gyri ( 10\%-15\%) (Weiskrantz and Saunders, 1984; Buckley et al., 1997). We speculate that the combination of being seizure-free and the striking stability of the rest of the face network after resection likely contributed to the fact that Patient S.P.'s behavioral performance did not worsen after removal of IOG-faces/OFA and posterior portion of pFus-faces/FFA-1.

Nevertheless, we cannot rule out the possibility that other, more difficult, face tasks may have revealed a further impairment in Patient S.P. after resection or that additional neural changes may have occurred within smaller temporal or spatial scales than those presently measured. At least two alternatives are possible: (1) short-term changes may occur immediately (e.g., seconds to hours) following resection after which the organization recovers within days or weeks; or (2) brain changes may occur at the submillimeter level, and cannot be detected with fMRI measurements at the millimeter and centimeter scale. Future studies will test these alternatives and further uncover the different spatial and temporal scales of cortical resiliency following focal damage.

\section{Confronting limitations in neuroimaging case studies}

We acknowledge that our case has two main limitations. Before surgery, Patient S.P. had (1) chronic seizures, and (2) aspects of her individual face discrimination were below normal, but above chance. These two limitations raise the question whether Patient S.P.'s brain was impaired before surgery. To address these limitations, we compared her face network before surgery with healthy controls. Our data show that the presurgery localization, selectivity, and variability of her face network and, the IOG in particular, were not different from these control participants. Nonetheless, it is possible that our experimental manipulations and metrics were not sensitive enough and that more sensitive manipulations, such as fMRI adaptation (Grill-Spector and Malach, 2001; Schiltz et al., 2006; Fox et al., 2013), may have identified subtle changes in her face network. An alternative possibility is that her behavioral impairment is due to brain changes outside the "core" face network as has been observed in developmental/ congenital prosopagnosics (Behrmann et al., 2007; Avidan and Behrmann, 2009; Garrido et al., 2009). Critically, we prioritized test-retest reliability across sessions before surgery rather than conducting additional measurements to establish a betweensession baseline relative to which we could determine the effects of surgery on cortical responses.

Despite these limitations, we believe that our case provides important causal evidence for a multiple route face network with nonhierarchical components. As there is only one other documented case with pre-resection and post-resection neuroimaging measurements (Gaillard et al., 2006), the present gold standard of causal tests in the field is neuropsychological case studies of patients with long-term brain damage. While these cases provide invaluable insights into the causal effects of cortical damage to perception, there are also limitations to these studies. For example, the brain damage in tested patients is often large and nonspe- 
cific and can encompass both hemispheres, the timescale from damage to measurement is long (on the order of years), and finally, each case only has a functional measurement after, but not before, damage has occurred. Because of these limitations, it is unknown exactly which aspects of the brain and behavior were typical before the damage (Marotta et al., 2001; Rossion et al., 2003; Schiltz et al., 2006; Steeves et al., 2006; Sorger et al., 2007; Rossion, 2008; Fox et al., 2011, 2013; Konen et al., 2011; Yang et al., 2016). Nevertheless, despite the different approaches between the present case and these prior cases in patients with long-term brain damage, it is reassuring that the results converge toward consistent conclusions of a multiple route hierarchy of cortical face processing supporting face perception.

In conclusion, our unique data provide causal insight into the construction of functional networks in the human brain and provide empirical support for recent neurocognitive models proposing a multiple route cortical network with nonhierarchical components underlying face processing.

\section{References}

Afraz A, Boyden ES, DiCarlo JJ (2015) Optogenetic and pharmacological suppression of spatial clusters of face neurons reveal their causal role in face gender discrimination. Proc Natl Acad Sci U S A 112:6730-6735. CrossRef Medline

Ahissar M, Hochstein S (2004) The reverse hierarchy theory of visual perceptual learning. Trends Cogn Sci 8:457-464. CrossRef Medline

Ajina S, Pestilli F, Rokem A, Kennard C, Bridge H (2015) Human blindsight is mediated by an intact geniculo-extrastriate pathway. Elife 4:piiee08935. CrossRef Medline

Allison T, McCarthy G, Nobre A, Puce A, Belger A (1994) Human extrastriate visual cortex and the perception of faces, words, numbers, and colors. Cereb Cortex 4:544-554. CrossRef Medline

Amano K, Wandell BA, Dumoulin SO (2009) Visual field maps, population receptive field sizes, and visual field coverage in the human $\mathrm{MT}^{+}$complex. J Neurophysiol 102:2704-2718. CrossRef Medline

Atkinson AP, Adolphs R (2011) The neuropsychology of face perception: beyond simple dissociations and functional selectivity. Philos Trans R Soc Lond B Biol Sci 366:1726-1738. CrossRef Medline

Avidan G, Behrmann M (2009) Functional MRI reveals compromised neural integrity of the face processing network in congenital prosopagnosia. Curr Biol 19:1146-1150. CrossRef Medline

Bancaud J, Talairach J (1973) [Methodology of stereo EEG exploration and surgical intervention in epilepsy]. Rev Otoneuroophtalmol 45:315-328. Medline

Behrmann M, Avidan G, Gao F, Black S (2007) Structural imaging reveals anatomical alterations in inferotemporal cortex in congenital prosopagnosia. Cereb Cortex 17:2354-2363. CrossRef Medline

Benson NC, Butt OH, Datta R, Radoeva PD, Brainard DH, Aguirre GK (2012) The retinotopic organization of striate cortex is well predicted by surface topology. Curr Biol 22:2081-2085. CrossRef Medline

Benson NC, Butt OH, Brainard DH, Aguirre GK (2014) Correction of distortion in flattened representations of the cortical surface allows prediction of V1-V3 functional organization from anatomy. PLoS Comput Biol 10: e1003538. CrossRef Medline

Benton AL, Sivan AB, Hamsher K, Varney NR, Spreen O (1983) Benton facial recognition: stimulus and multiple choice pictures. Lutz, FL: Psychological Assessment Resources.

Bernstein M, Yovel G (2015) Two neural pathways of face processing: a critical evaluation of current models. Neurosci Biobehav Rev 55:536-546. CrossRef Medline

Bertini G, Buffalo EA, De Weerd P, Desimone R, Ungerleider LG (2004) Visual responses to targets and distracters by inferior temporal neurons after lesions of extrastriate areas V4 and TEO. Neuroreport 15:1611-1615. CrossRef Medline

Bouvier SE, Engel SA (2006) Behavioral deficits and cortical damage loci in cerebral achromatopsia. Cereb Cortex 16:183-191. CrossRef Medline

Buckley MJ, Gaffan D, Murray EA (1997) Functional double dissociation between two inferior temporal cortical areas: perirhinal cortex versus middle temporal gyrus. J Neurophysiol 77:587-598. Medline

Buffalo EA, Bertini G, Ungerleider LG, Desimone R (2005) Impaired filter- ing of distracter stimuli by TE neurons following V4 and TEO lesions in macaques. Cereb Cortex 15:141-151. CrossRef Medline

Bullier J (2001) Integrated model of visual processing. Brain Res Brain Res Rev 36:96-107. CrossRef Medline

Busigny T, Joubert S, Felician O, Ceccaldi M, Rossion B (2010) Holistic perception of the individual face is specific and necessary: evidence from an extensive case study of acquired prosopagnosia. Neuropsychologia 48:4057-4092. CrossRef Medline

Crawford JR, Howell DC (1998) Comparing an individual's test score against norms derived from small samples. Clin Neuropsychol 12 : 482-486. CrossRef

Dale AM, Fischl B, Sereno MI (1999) Cortical surface-based analysis: I. Segmentation and surface reconstruction. Neuroimage 9:179-194. CrossRef Medline

Desimone R, Ungerleider LG (1986) Multiple visual areas in the caudal superior temporal sulcus of the macaque. J Comp Neurol 248:164-189. CrossRef Medline

Duchaine B, Nakayama K (2006) The Cambridge Face Memory Test: results for neurologically intact individuals and an investigation of its validity using inverted face stimuli and prosopagnosic participants. Neuropsychologia 44:576-585. CrossRef Medline

Duchaine B, Yovel G (2015) A revised neural framework for face processing. Annu Rev Vis Sci 1:393-416. CrossRef

Engel SA, Glover GH, Wandell BA (1997) Retinotopic organization in human visual cortex and the spatial precision of functional MRI. Cereb Cortex 7:181-192. CrossRef Medline

Fairhall SL, Ishai A (2007) Effective connectivity within the distributed cortical network for face perception. Cereb Cortex 17:2400-2406. CrossRef Medline

Felleman DJ, Van Essen DC (1991) Distributed hierarchical processing in the primate cerebral cortex. Cereb Cortex 1:1-47. CrossRef Medline

Fox CJ, Iaria G, Barton JJ (2008) Disconnection in prosopagnosia and face processing. Cortex 44:996-1009. CrossRef Medline

Fox CJ, Hanif HM, Iaria G, Duchaine BC, Barton JJ (2011) Perceptual and anatomic patterns of selective deficits in facial identity and expression processing. Neuropsychologia 49:3188-3200. CrossRef Medline

Fox CJ, Iaria G, Duchaine BC, Barton JJ (2013) Residual fMRI sensitivity for identity changes in acquired prosopagnosia. Front Psychol 4:756. CrossRef Medline

Gaillard R, Naccache L, Pinel P, Clémenceau S, Volle E, Hasboun D, Dupont S, Baulac M, Dehaene S, Adam C, Cohen L (2006) Direct intracranial, FMRI, and lesion evidence for the causal role of left inferotemporal cortex in reading. Neuron 50:191-204. CrossRef Medline

Garrido L, Furl N, Draganski B, Weiskopf N, Stevens J, Tan GC, Driver J, Dolan RJ, Duchaine B (2009) Voxel-based morphometry reveals reduced grey matter volume in the temporal cortex of developmental prosopagnosics. Brain 132:3443-3455. CrossRef Medline

Gauthier I, Skudlarski P, Gore JC, Anderson AW (2000) Expertise for cars and birds recruits brain areas involved in face recognition. Nat Neurosci 3:191-197. CrossRef Medline

Goffaux V, Peters J, Haubrechts J, Schiltz C, Jansma B, Goebel R (2011) From coarse to fine? Spatial and temporal dynamics of cortical face processing. Cereb Cortex 21:467-476. CrossRef Medline

Golarai G, Ghahremani DG, Whitfield-Gabrieli S, Reiss A, Eberhardt JL, Gabrieli JD, Grill-Spector K (2007) Differential development of highlevel visual cortex correlates with category-specific recognition memory. Nat Neurosci 10:512-522. CrossRef Medline

Gomez J, Pestilli F, Witthoft N, Golarai G, Liberman A, Poltoratski S, Yoon J, Grill-Spector K (2015) Functionally defined white matter reveals segregated pathways in human ventral temporal cortex associated with category-specific processing. Neuron 85:216-227. CrossRef Medline

Grill-Spector K, Malach R (2001) fMR-adaptation: a tool for studying the functional properties of human cortical neurons. Acta Psychol (Amst) 107:293-321. CrossRef Medline

Gschwind M, Pourtois G, Schwartz S, Van De Ville D, Vuilleumier P (2012) White matter connectivity between face-responsive regions in the human brain. Cereb Cortex 22:1564-1576. CrossRef Medline

Haxby JV, Hoffman EA, Gobbini MI (2000) The distributed human neural system for face perception. Trends Cogn Sci 4:223-233. CrossRef Medline

Hemond CC, Kanwisher NG, Op de Beeck HP (2007) A preference for contralateral stimuli in human object- and face-selective cortex. PLoS One 2:e574. CrossRef Medline 
Hermes D, Miller KJ, Noordmans HJ, Vansteensel MJ, Ramsey NF (2010) Automated electrocorticographic electrode localization on individually rendered brain surfaces. J Neurosci Methods 185:293-298. CrossRef Medline

Heywood CA, Cowey A (1992) The role of the 'face-cell' area in the discrimination and recognition of faces by monkeys. Philos Trans R Soc Lond B Biol Sci 335:31-37; discussion 37-38. CrossRef Medline

Jacques C, Witthoft N, Weiner KS, Foster BL, Rangarajan V, Hermes D, Miller KJ, Parvizi J, Grill-Spector K (2016) Corresponding ECoG and fMRI category-selective signals in human ventral temporal cortex. Neuropsychologia 83:14-28. CrossRef Medline

Jiang F, Dricot L, Weber J, Righi G, Tarr MJ, Goebel R, Rossion B (2011) Face categorization in visual scenes may start in a higher order area of the right fusiform gyrus: evidence from dynamic visual stimulation in neuroimaging. J Neurophysiol 106:2720-2736. CrossRef Medline

Jonas J, Descoins M, Koessler L, Colnat-Coulbois S, Sauvée M, Guye M, Vignal JP, Vespignani H, Rossion B, Maillard L (2012) Focal electrical intracerebral stimulation of a face-sensitive area causes transient prosopagnosia. Neuroscience 222:281-288. CrossRef Medline

Kanwisher N, McDermott J, Chun MM (1997) The fusiform face area: a module in human extrastriate cortex specialized for face perception. J Neurosci 17:4302-4311. Medline

Kay KN, Weiner KS, Grill-Spector K (2015) Attention reduces spatial uncertainty in human ventral temporal cortex. Curr Biol 25:595-600. CrossRef Medline

Kim M, Ducros M, Carlson T, Ronen I, He S, Ugurbil K, Kim DS (2006) Anatomical correlates of the functional organization in the human occipitotemporal cortex. Magn Reson Imaging 24:583-590. CrossRef Medline

Konen CS, Behrmann M, Nishimura M, Kastner S (2011) The functional neuroanatomy of object agnosia: a case study. Neuron 71:49-60. CrossRef Medline

Kravitz DJ, Saleem KS, Baker CI, Ungerleider LG, Mishkin M (2013) The ventral visual pathway: an expanded neural framework for the processing of object quality. Trends Cogn Sci 17:26-49. CrossRef Medline

Lohse M, Garrido L, Driver J, Dolan RJ, Duchaine BC, Furl N (2016) Effective connectivity from early visual cortex to posterior occipitotemporal face areas supports face selectivity and predicts developmental prosopagnosia. J Neurosci 36:3821-3828. CrossRef Medline

Marotta JJ, Genovese CR, Behrmann M (2001) A functional MRI study of face recognition in patients with prosopagnosia. Neuroreport 12:15811587. CrossRef Medline

Mumford D (1992) On the computational architecture of the neocortex: II. The role of cortico-cortical loops. Biol Cybern 66:241-251. CrossRef Medline

Murphy K, Bodurka J, Bandettini PA (2007) How long to scan? The relationship between fMRI temporal signal to noise ratio and necessary scan duration. Neuroimage 34:565-574. CrossRef Medline

Op de Beeck HP, Baker CI, DiCarlo JJ, Kanwisher NG (2006) Discrimination training alters object representations in human extrastriate cortex. J Neurosci 26:13025-13036. CrossRef Medline

O’Toole AJ, Roark DA, Abdi H (2002) Recognizing moving faces: a psychological and neural synthesis. Trends Cogn Sci 6:261-266. CrossRef Medline

Palermo R, Rossion B, Rhodes G, Laguesse R, Tez T, Hall B, Albonico A, Malaspina M, Daini R, Irons J, Al-Janabi S, Taylor LC, Rivolta D, McKone E (2016) Do people have insight into their face recognition abilities? Q J Exp Psychol (Hove) 23:1-16. CrossRef Medline

Parvizi J, Jacques C, Foster BL, Withoft N, Rangarajan V, Weiner KS, GrillSpector K (2012) Electrical stimulation of human fusiform faceselective regions distorts face perception. J Neurosci 32:14915-14920. CrossRef Medline

Pasternak O, Sochen N, Gur Y, Intrator N, Assaf Y (2009) Free water elimination and mapping from diffusion MRI. Magn Reson Med 62:717-730. CrossRef Medline

Pestilli F, Yeatman JD, Rokem A, Kay KN, Wandell BA (2014) Evaluation and statistical inference for human connectomes. Nat Methods 11:10581063. CrossRef Medline

Pinsk MA, Arcaro M, Weiner KS, Kalkus JF, Inati SJ, Gross CG, Kastner S (2009) Neural representations of faces and body parts in macaque and human cortex: a comparative FMRI study. J Neurophysiol 101:25812600. CrossRef Medline

Pitcher D, Walsh V, Yovel G, Duchaine B (2007) TMS evidence for the involvement of the right occipital face area in early face processing. Curr Biol 17:1568-1573. CrossRef Medline

Pitcher D, Walsh V, Duchaine B (2011a) The role of the occipital face area in the cortical face perception network. Exp Brain Res 209:481-493. CrossRef Medline

Pitcher D, Dilks DD, Saxe RR, Triantafyllou C, Kanwisher N (2011b) Differential selectivity for dynamic versus static information in face-selective cortical regions. Neuroimage 56:2356-2363. CrossRef Medline

Pitcher D, Duchaine B, Walsh V (2014) Combined TMS and FMRI reveal dissociable cortical pathways for dynamic and static face perception. Curr Biol 24:2066-2070. CrossRef Medline

Puce A, Allison T, Gore JC, McCarthy G (1995) Face-sensitive regions in human extrastriate cortex studied by functional MRI. J Neurophysiol 74:1192-1199. Medline

Puce A, Allison T, Bentin S, Gore JC, McCarthy G (1998) Temporal cortex activation in humans viewing eye and mouth movements. J Neurosci 18:2188-2199. Medline

Pyles JA, Verstynen TD, Schneider W, Tarr MJ (2013) Explicating the face perception network with white matter connectivity. PLoS One 8:e61611. CrossRef Medline

Rossion B (2008) Constraining the cortical face network by neuroimaging studies of acquired prosopagnosia. Neuroimage 40:423-426. CrossRef Medline

Rossion B, Caldara R, Seghier M, Schuller AM, Lazeyras F, Mayer E (2003) A network of occipito-temporal face-sensitive areas besides the right middle fusiform gyrus is necessary for normal face processing. Brain 126:23812395. CrossRef Medline

Rossion B, Dricot L, Goebel R, Busigny T (2011) Holistic face categorization in higher order visual areas of the normal and prosopagnosic brain: toward a non-hierarchical view of face perception. Front Hum Neurosci 4:225. CrossRef Medline

Rossion B, Hanseeuw B, Dricot L (2012) Defining face perception areas in the human brain: a large-scale factorial fMRI face localizer analysis. Brain Cogn 79:138-157. CrossRef Medline

Scherf KS, Behrmann M, Humphreys K, Luna B (2007) Visual category selectivity for faces, places and objects emerges along different developmental trajectories. Dev Sci 10:F15-F30. CrossRef Medline

Schiltz C, Sorger B, Caldara R, Ahmed F, Mayer E, Goebel R, Rossion B (2006) Impaired face discrimination in acquired prosopagnosia is associated with abnormal response to individual faces in the right middle fusiform gyrus. Cereb Cortex 16:574-586. CrossRef Medline

Sergent J, Ohta S, MacDonald B (1992) Functional neuroanatomy of face and object processing: a positron emission tomography study. Brain 115: 15-36. CrossRef Medline

Sorger B, Goebel R, Schiltz C, Rossion B (2007) Understanding the functional neuroanatomy of acquired prosopagnosia. Neuroimage 35: 836-852. CrossRef Medline

Steeves JK, Culham JC, Duchaine BC, Pratesi CC, Valyear KF, Schindler I, Humphrey GK, Milner AD, Goodale MA (2006) The fusiform face area is not sufficient for face recognition: evidence from a patient with dense prosopagnosia and no occipital face area. Neuropsychologia 44:594-609. CrossRef Medline

Steeves J, Dricot L, Goltz HC, Sorger B, Peters J, Milner AD, Goodale MA, Goebel R, Rossion B (2009) Abnormal face identity coding in the middle fusiform gyrus of two brain-damaged prosopagnosic patients. Neuropsychologia 47:2584-2592. CrossRef Medline

Stigliani A, Weiner KS, Grill-Spector K (2015) Temporal processing capacity in high-level visual cortex is domain-specific. J Neurosci 35:1241212424. CrossRef Medline

Takemura H, Rokem A, Winawer J, Yeatman JD, Wandell BA, Pestilli F (2016) A major human white matter pathway between dorsal and ventral visual cortex. Cereb Cortex 26:2205-2214. CrossRef Medline

Tavor I, Yablonski M, Mezer A, Rom S, Assaf Y, Yovel G (2014) Separate parts of occipito-temporal white matter fibers are associated with recognition of faces and places. Neuroimage 86:123-130. CrossRef Medline

Tournier JD, Calamante F, Connelly A (2007) Robust determination of the fibre orientation distribution in diffusion MRI: non-negativity constrained super-resolved spherical deconvolution. Neuroimage 35:14591472. CrossRef Medline

Tournier JD, Calamante F, Connelly A (2012) MRtrix: diffusion tractography in crossing fibre regions. Int J Imaging Syst Technol 22:53-66. CrossRef 
Turk-Browne NB, Norman-Haignere SV, McCarthy G (2010) Face-specific resting functional connectivity between the fusiform gyrus and posterior superior temporal sulcus. Front Hum Neurosci 4:176. CrossRef Medline

Ungerleider LG, Mishkin M (1982) Two cortical visual systems. In: Analysis of visual behavior (Ingle DJ, Goodale MA, Mansfield RJW, eds), pp 549-586. Cambridge, MA: Massachusetts Institute of Technology.

Wandell BA, Smirnakis SM (2009) Plasticity and stability of visual field maps in adult primary visual cortex. Nat Rev Neurosci 10:873-884. CrossRef Medline

Wandell BA, Chial S, Backus BT (2000) Visualization and measurement of the cortical surface. J Cogn Neurosci 12:739-752. CrossRef Medline

Warner CE, Goldshmit Y, Bourne JA (2010) Retinal afferents synapse with relay cells targeting the middle temporal area in the pulvinar and lateral geniculate nuclei. Front Neuroanat 4:8. CrossRef Medline

Warrington EK, James M (1991) The Visual Object and Space Perception Battery. Bury St Edmunds, England: Thames Valley Test.

Weiner KS, Grill-Spector K (2010) Sparsely-distributed organization of face and limb activations in human ventral temporal cortex. Neuroimage 52: 1559-1573. CrossRef Medline

Weiner KS, Grill-Spector K (2011) Not one extrastriate body area: using anatomical landmarks, $\mathrm{hMT}^{+}$, and visual field maps to parcellate limbselective activations in human lateral occipitotemporal cortex. Neuroimage 56:2183-2199. CrossRef Medline

Weiner KS, Grill-Spector K (2013) Neural representations of faces and limbs neighbor in human high-level visual cortex: evidence for a new organization principle. Psychol Res 77:74-97. CrossRef Medline
Weiner KS, Golarai G, Caspers J, Chuapoco MR, Mohlberg H, Zilles K, Amunts K, Grill-Spector K (2014) The mid-fusiform sulcus: a landmark identifying both cytoarchitectonic and functional divisions of human ventral temporal cortex. Neuroimage 84:453-465. CrossRef Medline

Weiner KS, Yeatman JD, Wandell BA (2016) The posterior arcuate fasciculus and the vertical occipital fasciculus. Cortex. Advance online publication. Retrieved Mar. 31, 2016. doi: 10.1016/j.cortex.2016.03.012. CrossRef Medline

Weiskrantz L, Saunders RC (1984) Impairments of visual object transforms in monkeys. Brain 107:1033-1072. CrossRef Medline

Yang H, Susilo T, Duchaine B (2016) The anterior temporal face area contains invariant representations of face identity that can persist despite the loss of right FFA and OFA. Cereb Cortex 26:1096-1107. CrossRef Medline

Yeatman JD, Weiner KS, Pestilli F, Rokem A, Mezer A, Wandell BA (2014) The vertical occipital fasciculus: a century of controversy resolved by in vivo measurements. Proc Natl Acad Sci US A 111:E5214-E5223. CrossRef Medline

Zhen Z, Fang H, Liu J (2013) The hierarchical brain network for face recognition. PLoS One 8:e59886. CrossRef Medline

Zhen Z, Yang Z, Huang L, Kong XZ, Wang X, Dang X, Huang Y, Song Y, Liu J (2015) Quantifying interindividual variability and asymmetry of faceselective regions: a probabilistic functional atlas. Neuroimage 113:13-25. CrossRef Medline

Zhu Q, Zhang J, Luo YL, Dilks DD, Liu J (2011) Resting-state neural activity across face-selective cortical regions is behaviorally relevant. J Neurosci 31:10323-10330. CrossRef Medline 